\title{
Properties, categories, and categorisation
}

\author{
Sébastien Poitrenaud, Jean-François Richard, and \\ Charles Tijus \\ Université Paris VIII, France
}

\begin{abstract}
We re-evaluate existing data that demonstrate a large amount of variability in the content of categories considering the fact that these data have been obtained in a specific task: the production of features of single isolated categories. We present new data that reveal a large consensus when participants have to judge whether or not a given feature is characteristic of a category and we show that classification tasks produce an intermediate level of consensus. We argue that the differences observed between tasks are due to the extent of implied context and we propose a reinterpretation of typicality effects, demonstrating that they are compatible with the existence of a stable conceptual core. In order to explain how the existence of a conceptual core is consistent with variability due to context, we present a theory of categorisation based on a property tree organisation. Within a domain of description, we distinguish between semantic implications (flying $\rightarrow$ moving) and empirical implications (flying $\rightarrow$ having wings) as well as between properties used to describe objects. Semantic implications serve to build property lines and Galois lattices are used to reveal category structures according to empirical implications. We show that variability in the category content may be explained by the fact that some properties are emphasised while others are masked according to the context.
\end{abstract}

\section{INTRODUCTION}

The role and nature of categories, as well as the process of categorisation itself, are all highly controversial. The last 40 years have been marked by a succession of theories. They were widely accepted for a time, then their very foundations were challenged, and finally they were replaced by new conceptions based on opposite premises.

Beyond doubt, these controversies reflect an oscillation between empiricism and rationalism. However it is equally obvious that these

Correspondence should be addressed to Sébastien Poitrenaud, CNRS-Université Paris 8, 2 rue de la Liberté, 93526 Saint-Denis, France.

(C) 2005 Psychology Press Ltd

http://www.tandf.co.uk/journals/pp/13546783.html

DOI: $10.1080 / 13546780442000169$ 
oscillations have been motivated by empirical results that have seemed incompatible within the existing frameworks. To be valid, any theory that is put forward must explain the entire body of firmly established experimental results. So far, each theory has been supported by a specific set of data, whereas the whole set of empirical results has never been fully integrated into a single theoretical framework. In our opinion, we need to re-examine some basic concepts, such as the nature of a property and consequently the nature of a category that, until now, have never been precise enough to enable researchers to discuss the significance of available data with regard to competing theories. We also need to examine the theoretical significance of the effects of context. The aim of this paper is to propose a re-examination of these basic issues that will allow a reinterpretation of available data and a re-evaluation of existing positions. Two main questions have been addressed: What is the content of a natural category? And, does a conceptual core exist?

\section{The content of natural categories}

The classical theory of concepts has been rejected on empirical grounds. The most important of these is probably the existence of typicality effects, as demonstrated by the work of Rosch. This effect was highlighted in a variety of tasks: evaluation of the typicality of items by participants (Rosch, 1975a), frequency and order of item output when participants are invited to produce exemplars of categories (Rosch, 1975b), verification time for category membership (Rosch, Mervis, Gray, Johnson, \& Boyes-Braem, 1976), and priming of a category by an exemplar (Rosch et al., 1976). This effect is regarded as a definitive argument against the idea that concepts are defined by the possession of criterial properties, since it contradicts one immediate consequence of this idea, namely that all exemplars of a concept should be equivalent. This criticism was formulated by Rosch (e.g., 1975a, 1975b) and has been acknowledged by most authors since.

Referring to the notion of family resemblance, introduced by Wittgenstein (1953), Rosch and Mervis (1975) proposed a measure of family resemblance based on the mean number of properties shared by each exemplar with all the members of the category. Each property was weighted in function of the frequency with which it was attributed. Thus, category membership does not depend on the ownership of a set of shared properties but on the evaluation of the resemblance of the candidate to a particular exemplar, clearest case, or best example of the category, called the prototype, or to a set of these exemplars. In a category centred on a prototype, the exemplars are not equivalent: they may be ordered on a typicality scale that reflects their degree of resemblance to the prototype. Consequently, some members lie at the centre of the category, while others are located on its 
boundaries. Overall, however, all the members share a family resemblance that is due to the distribution of the most frequent properties of those members.

Among the different objections raised to this theory, probably the most serious arises from the basic assumption of family resemblance theory, namely the likening of the decision of category membership to a judgement of similarity, and is undermined by data showing that similarity judgement is dependent on context (Medin, Goldstone, \& Gentner, 1993; Tversky, 1977), or that there was dissociation between similarity and the decision of category membership (see Rips, 1989, for a review). Being asked to imagine a hypothetical object 3 inches in diameter and to compare it with a quarter or a pizza, participants judge it to be more similar to a quarter but more likely to be a pizza, because the size is known to be fixed for quarters but variable for pizzas.

\section{The question of conceptual core}

In response to these difficulties, an alternative to the prototype view progressively emerged, promoted by the influential paper by Murphy and Medin (1985). They considered that the basic characteristic of categories is conceptual coherence and that this coherence reflects a theory about what kind of thing is referred to by the category. This position differs from the classical view, in that the emphasis is not on the definitional aspect of properties but on the relations between them: a concept is not a list of properties, because properties are linked to each other by an intrinsic coherence that constitutes a kind of essence. What matters is not how things are in reality, rather it is people's conception of them. Medin and Ortony (1989) speak of psychological essentialism, taking as a basic fact the idea that people believe that things have essences. Relations between properties must not be considered merely as empirical correlations. These relations are meaningful because most of them are causal in nature. For instance, we know that both birds and insects have wings and they can both fly. Because they can fly, birds are also able to catch and eat insects. We know that mammals suckle their young but that birds do not, because birds lay eggs.

The idea that concepts are stable and fundamentally nonperceptual has been challenged by a group of authors on the basis that the structures which control behaviour are temporary representations constructed in the specific contexts related to the tasks and that, as it has not been demonstrated that conceptual cores affect behaviour, there is no need for them. As Barsalou (1993a) said, "Until we have evidence that stable conceptual cores in longterm memory control behavior in some task, we have little reason to believe in their existence" (pp. 175-176). Jones and Smith (1993) proposed that "the perceived similarity of objects is emergent and determined by the joint 
effect of multiple kinds of forces" (p. 134). Finally, concepts do not exist in long-term memory, but are assembled on-line from multiple knowledge sources and specific experience provided by the task context.

The basis for this position is the degree of variability and instability in category representations, in tasks of both property generation and judgement of typicality, demonstrated by Barsalou in a series of influential studies (Barsalou, 1987; Barsalou \& Medin, 1986; see Barsalou, 1989, for a summary). There is a high degree of variability across individuals: in property generation, the overlap of a participant's description with another participant's description for the same category is about one-third. There is also a great deal of variability among the descriptions given by the same participants when they are invited to adopt different points of views: their own point of view, the housewife's point of view, and a Chinese person's point of view. Moreover, when the same participants repeat the production task a few days or a few weeks later, a large degree of variability can be observed in the protocols of each given participant. In data collected by Barsalou (1989), the overlap of two protocols of the same participant for two different sessions for the same category was a little more than half. Bellezza (1984) and Vrignaud (1998) reported similar results. Moreover, many categories are ad hoc categories, such as things to bring for a picnic, which are related to goals and for this reason are dependent on the context of the task.

The aim of this paper is to put forward proposals in order to answer the difficult question of how stable representations produce variability and how this variability is adaptive. We propose a new version of the conceptual core view that is related both to classical theory and to conceptual coherence theory, but which introduces new conceptions about the nature of properties involved in the delimitation of categories and about the role of context. Our aim is to explore: (i) the semantic content of categories which allows for the idea that judgement for category membership entails prediction of properties not observed but known to belong to the category, (ii) their hierarchical organisation, based on inclusion relations, which explains deductive inferences, (iii) the emergence of typicality effects, and (iv) the effect of context responsible for the variability observed in effective categorisation behaviour.

The gist of our argument is as follows. As far as the nature of properties is concerned, although the importance of relations between properties has been stressed (e.g., Malt, 1995), the only relations that have been considered are either causal (having wings and flying) or correlational (having feathers and having a beak) in nature. We want to emphasise the importance of another type of relation that, though present in the attribute/value distinction, has not been considered in its full scope. This is the semantic implication that, at the intentional level, is the counterpart of the inclusion 
relation at the extensional level. We will argue that this relation is not limited to two levels but is fundamental to the definition of a property: it defines a series of levels regarding the dimension of generality/specificity, e.g., vermilion, red, coloured. This allows a property to be considered at a more or less specific level and this is, in our view, responsible for the fact that an object may be named at different levels and, more generally, for the hierarchical organisation of categories.

As regards context, its effect has been widely recognised at the empirical level but, with the exception of certain authors (e.g., Barsalou), its role has not been regarded as central in the definition of the categorisation process. We will argue that variability is constitutive of categorisation, because categorisation always takes place in a given context, characterised by the elements of the situation, the participant's semantic knowledge, and the goal $\mathrm{s} / \mathrm{he}$ has at the time. In this way, what is meant by a category name will differ according to the context. It is possible that emergent properties simply appear, as in the case of conceptual combination (see Hampton, 1993) or of metaphor (Tourangeau \& Rips, 1991). However, this does not mean that categories are not delimited by properties. Depending on the context, only a subset of properties is activated, that which is shared with the elements of the context and therefore is relevant to it. It follows that the meaning of the category may differ somewhat, although the potential set of properties defining it, which constitutes what is known about the category, is quite stable, at least in a given culture and at a given level of development.

\section{Recent evidence for the existence of a conceptual core}

The claim of the non-existence of a conceptual core has been defended both by the advocates of the prototype theory (e.g., Rosch, 1975a; Rosch \& Mervis, 1975) and by authors who emphasise the effect of context (Barsalou, 1993a; Jones \& Smith, 1993). This claim is based on a substantial set of empirical results, those produced by the seminal study by Rosch and Mervis (1975) and those demonstrating category variability (Barsalou, 1989). The question is how best to interpret these results. We will present two arguments in favour of the idea of the existence of a conceptual core: the first is empirical and the second theoretical.

Recently, new data have appeared suggesting that the existing evidence concerning the degree of agreement about the content of categories has to be re-examined. One concern is that the results advanced as proof that categories have no conceptual content (as opposed to those advanced as proofs of typicality) have all been observed in the same situation, namely a task in which participants are shown subcategory labels one at a time, and are asked to generate properties that are characteristic of that class of objects. The fact is that very few properties, if any, are common to all the 
subcategories of a given category. How is this fact to be interpreted? Does this mean that participants ignore generic properties or that, when asked to list "the characteristics and attributes that people feel are common to and characteristic of different kinds of ordinary everyday objects" (Rosch \& Mervis, 1975, p. 578), participants understand that they are expected to give the distinctive properties of the mentioned type of entity?

We would claim that these types of instructions may lead participants to give priority to the production of responses at a specific level, that is to say at a level that emphasises contrasts with collateral categories. This effect is due to pragmatic factors such as informativity maxims (Grice, 1975; Sperber \& Wilson, 1986), which lead people to apply conversation rules, to be as informative as possible, and thus to express themselves at a rather specific level; a level where distinctive properties are used to contrast a category with its neighbours. For example if we take the question "What are the common characteristic properties of apples?" participants may be found to provide answers more related to the question "What kind of fruit are apples?". When this is the case, their answers would implicitly be in the form: "An apple is a fruit which ...". Answering this kind of question does not require us to mention the shared properties of fruit. Indeed, if we say or imply that an apple is a fruit, we do not need to list the properties of fruit because mentioning the name of the category serves as an implicit reference to the shared properties of named objects.

Actually, to conclude that this task entails focusing on distinctive properties may be a logical extension of our theory of the effects of context and we will give a detailed presentation of this concept later in this paper. In our view, when the participant is shown a single subcategory and asked to list the properties of that subcategory, in this limited context, only the distinctive properties within the category will be activated, not those properties that serve to distinguish between that particular category and contrasting ones. For instance the word cherry will not activate the properties that set fruits apart from vegetables, such as how they grow, when they are gathered, how and when they are eaten, which are properties common to fruits and distinctive of vegetables. A fortiori, it will not evoke properties that are common to fruits and vegetables alike, such as eaten by humans. The properties that will be activated are is red, has juice, has a stone, stains, etc.

We can expect that less distinctive properties will be generated when the context is larger, namely when two or more category labels are presented. A larger number of properties will be activated, as this set of properties will include not only the distinctive properties of each subcategory, but also the properties that are shared by the two subcategories and belong to the common category. Vrignaud (1998) compared the number of common properties listed by a participant when the subcategories were presented in 
pairs with the number of properties common to the same two subcategories listed by the same participant when the two subcategory terms were presented in isolation. There was a month's interval between each session. When two subcategories (sparrow and eagle, for instance) were presented together, a mean of 3.35 properties were common to the superordinate category, compared with 0.78 when they were presented in isolation. This result supports the idea that when the subcategories are presented in isolation, the participants look for properties that are specific to the subcategories and overlook those that are shared with contrast subcategories. This effect, as Vrignaud suggests, is probably related to the structural alignment effect described by Markman and Gentner (1993b), whereby common properties which can be aligned because they are shared at a deeper level are favoured in a comparison task, whereas when similarities between two objects or two scenes are being evaluated, more superficial features are taken into account. This point will be discussed more thoroughly later.

When tasks other than enumerating the properties of objects are used in order to assess the cognitive content of categories, the degree of consensus appears substantially higher. Consider first the results obtained in classification tasks. Such tasks should produce still more common features than those observed by Vrignaud (1998). Since a large number of category labels are presented, more properties are therefore activated to encompass the categories present in this context. Intercultural studies reviewed by Malt (1995) and a later study by Lopez, Atran, Coley, and Medin (1997) highlighted a substantial level of agreement between participants of different cultures in the definition of natural categories. Similarly, the study by Medin, Lynch, Coley, and Atran (1997), which compared the classification of trees by three kinds of experts - taxonomists, maintenance personnel, and landscape gardeners - obtained a large agreement in the classification and verbalisation of properties characterising the different classes. The differences that did arise could easily be explained by differences in the types of properties considered, the landscapers lending greater importance to morphological and ornamental properties. In the same way the study by Ross and Murphy (1999) reveals a substantial agreement about the properties of various food items

Another interesting task is the judgement task where participants are asked to assess whether or not a given property belongs to a category. In our perspective, we would expect agreement to be greatest in a judgement task, since the effect of the context is ruled out in this task: the properties are stated and do not have to be evoked. Consistent with our predictions, perfect agreement between subjects is observed in this case. Vrignaud (1998) used a judgement task in which the categories were animal species including humans and the properties were biological properties (anatomical and behavioural) and mental properties. The only differences observed were 
related to the species and more specifically to their relative distance from humans. There were no inter-individual differences, whereas the same author in a property generation task observed massive inter-individual differences

A study was conducted in our laboratory (Héron-Banaicha, 1998), to test the hypothesis that the differences in results between a judgement task and the classical task of enumeration of the properties of a category are caused by differences in the availability of properties due to the context, and that if a conceptual core exists for natural categories, it should be manifest in a judgement task. Two conditions were compared: the property production task used by Rosch and Mervis (1975), and a judgement task in the form of a questionnaire, in which participants had to state whether or not each item possessed a given property. The items were subcategories of fruit (20) and vegetables (10) and the properties presented in the questionnaire concerned the appearance (colour, shape, size), the structure (parts), the way the item grew, the season when it was harvested, how and when it was eaten, its flavour, and the category to which it belonged. The questions were phrased either in a general way (it grows alone, it grows on something, it is eaten raw, it is eaten after preparation) or in a specific way (it grows on a tree, it grows on a bush, it is eaten as jam, it is eaten in tarts, it is eaten cooked).

The results of the production task replicated those obtained by Rosch and Mervis (1975). The only property mentioned by a majority of participants was the name of the category ( $73 \%$ for fruit and vegetables). The other properties differed highly between participants, the level of agreement being less than $20 \%$ for a property. Many of these properties were more specific than those that appeared in the questionnaire, for instance the characteristics of the flesh (mealy, firm, juicy, milky) or of the skin (thin, thick, smooth, rough, soft, sharp), the varieties of colours inside and outside, the nutritive value, the specific ways it is cooked, the instrument used to eat it (hand, spoon, fork). The mean number of properties mentioned for a subcategory by at least six participants was 2.33 . As a rule, few subjects mentioned the properties present in the questionnaire, but there were important differences according to the type of properties. We shall discuss this point later after presenting the data from the judgement task

In this last task, in contrast to the property generation task, the level of agreement was quite high, with 6.2 properties, as a mean for the 30 subcategories ( 20 fruits and 10 vegetables), recognised by each of the 10 participants as characteristic of a subcategory; 10.7 properties recognised as common by at least 9 participants and 16.1 by at least 7 participants.

Not only was there a high level of agreement for the subcategories but also the general categories of fruit and vegetable were assigned characteristic properties that are common to their subcategories. Vegetables grow by themselves, directly in the soil (.55 of the responses) while fruits grow on 
trees (.64) or bushes (.24). Vegetables have flesh (.68), leaves (.38), and skin (.39), and are of medium size (.70), while fruits have flesh (.90) skin (.64), pips (.34), stones (.24), and are small (.62) or medium (.41). Vegetables taste neutral (.65), are cooked and are eaten during main meals (.94 at lunch and 1.0 at dinner), while fruits taste sweet (.85) and can be eaten raw either outside meals (.90) or during meals (.74 at lunch, .84 at dinner). It is noticeable that when participants are unaware of a given property for a subcategory they may nevertheless respond and then attribute the property that is characteristic of the category. For instance, a number of participants answered that pineapples grow on trees. This is what is expected if a category is defined by a set of associated properties.

If we consider now the properties present in the questionnaire and compare how often they are evoked spontaneously and attributed in the questionnaire, we observe very important differences. The circumstances when fruits or vegetables are eaten are practically never mentioned in the generation task (less than .01), while the agreement is very high in the judgement task. The origin of food items is rarely evoked spontaneously, although it is evoked somewhat more frequently for vegetables (they grow on the ground .13 in the property generation task versus .55 in the judgement task) than for fruits (they grow on a tree .04 versus .63). Conversely size is mentioned somewhat more frequently for fruits (small .17 versus .62) than for vegetables (.08 versus .70). As regards the parts, the fact that fruits and vegetables are made of flesh, (which is recognised for both categories in the judgement task :.90 for fruits and .68 for vegetables) is rather rarely mentioned spontaneously (.08 and .11 respectively). On the other hand, leaves are mentioned rather frequently for vegetables (.20 versus .38 ), and specific parts of fruits are also evoked: skin (.22 against 64$)$, stone (.14 versus .24), pips (.10 versus .34). How food items taste is evoked spontaneously a bit more frequently for fruits (sweet .15 versus .83 ) than for vegetables (neutral .01 versus .65).

In summary, there is a big gap between what is known about food items and what is said in the property generation task. These results can be expected if one considers that in the production task the effect of context operates in the evocation of properties, while it is excluded in the judgement task. We shall see later how this effect of context may be explained. As our hypothesis predicts, the typical behaviour observed in the production task consisted in giving the name of the category, followed by very specific features. The production task generated distinctive characteristics, while the judgement task provided general knowledge about properties of the members of each subcategory. Clearly, once participants have said that an item is a fruit or a vegetable, they don't enumerate the characteristic properties of each category, although they know these properties, if we judge from the results of the judgement task. Hence we may conclude that 
the property generation task is not reliable for determining the cognitive content of categories.

The idea that information about a category is stored in long-term memory was put forward some years ago by Barsalou (1993a). When subjects are asked to provide definitions of categories such as birds, the overlap between subjects' definitions is only $44 \%$. However when the pool of features attributed to a category is presented and the subjects have to evaluate whether each property is a possible feature for some members of the category, very high agreement is observed. As Barsalou says, "Surprisingly, we found virtually perfect agreement both between and within subjects, with between-subject agreement being a 97\% overlap on average, and withinsubject agreement being 98\%" (Barsalou, 1993a, p. 33). As the author argues, this result shows that the variability demonstrated in the definition task does not reflect differences in the underlying knowledge in long-term memory, which instead appears highly similar for a given category.

As far as we know, this idea has not been followed up by other authors, probably because it was difficult to reconcile it from a theoretical point of view with the massive evidence of variability due to context effects and from an empirical point of view, with the seemingly conclusive evidence provided by the property generation task.

The purpose of the paper is threefold:

1 To challenge two widely accepted ideas: that typicality effects are an argument for the proposition that categories have fuzzy boundaries, by showing that typicality effects may be explained by the assignment of verbal labels to cognitive categories and hence rather reflect the meaning intended in a particular context than the true conceptual content of the category; and that the instability of category representations is not an argument against the existence of a conceptual core, because, as will be shown, the specific context of the task makes relevant only a subset of the properties of the cognitive category, which may differ among contexts.

2 To present a theory that assumes that the conceptual content of categories in memory is stable and proposes a mechanism for the categorisation process which selects the properties that are relevant for the context.

3 To provide an explanation for empirical results that are not in the scope of existing theories: the differences observed among the different tasks used to investigate the conceptual content of categories - there is an important variability between different individuals and even for the same individual at different times in the task of generation of properties, while there is a consensus when participants are asked to judge which properties in a list characterise a given category. The fact 
that a higher degree of agreement is observed in the property generation task when category labels are presented by pairs than when they are presented one at a time.

First we shall propose a new way of thinking about what a property is, showing that a property may be conceived at more than two levels of generality, as is done in the distinction between attribute and values. In a second step we shall use the Galois lattice formalism to show how categories are generated when stable sets of properties happen to be associated to sets of objects, and how typicality and cross-classification effects may be explained. Third, we shall show that the same formal mechanism explains categorisation in context, making salient some of the properties (and consequently some objects) due to the properties present in the other objects of the context and masking some other ones, and we shall discuss the implications of this position as regards the results mentioned above. The core of our argument is that it is the same mechanism that produces both stability and variability.

\section{THE STRUCTURE OF OBJECT PROPERTIES}

To understand why there are so many differences among these various tasks in terms of the number of properties attributed to a category, the nature of the properties that are elicited and the importance of inter-individual differences, we need to have a closer look at what a property is. What exactly do we mean by the properties of apples? "Grows on a plant", "used to make cider", "has a green colour" are, in various ways, all properties of apples. Categorisation behaviour produces property groupings that draw category outlines in many different ways, depending on chosen goals in a given context. Grows on a plant is a property of apples in so far as all apples grow on plants. This property belongs to the intension of the apple concept, that is to say it belongs to the intersection of the sets formed from the properties of each apple species. However, while it is indeed a "common" property of apples, it is not a distinctive (i.e., specific) property of apples, in so far as all fruits grow on plants. Used to make cider also belongs to the set of the shared properties of apples, but the part of the set which allows for contrast with fruits that are not apples. Cognitively, this kind of distinctive property is very useful because it is the only one that may safely be used to distinguish between apples and non-apples. Formally, these very specific properties are to be found in the complement of the intension of the fruit concept with regard to the intension of the apple concept. Has a yellow-red colour is a property of apples in so far as some familiar apples are yellow-red. This latter property is characteristic of some apple species and as such, is a candidate for the list of typical properties of apples. 
In order to discuss the theoretical implications of the preceding results in a more precise way, we present a theory featuring a mechanism for the selection of properties according to the objects present in the context and for the generation of ad hoc categories that are the most relevant in a given situation, i.e., categories that can adequately express similarities and differences between the objects present in the context.

What we call a property is the basic constituent of concept intension. Various kinds of properties may be distinguished: surface properties to qualify the appearance of objects, structural properties to describe the parts of objects and the physical matter they are made of. There are also functional properties, which correspond to know-how related to the use of objects. Regardless of these different types of properties, we are now going to focus on their internal organisation and structure.

\section{Traits vs attributes}

Traits and attributes are the main two structures used for properties. Traits are unary descriptors that serve to denote the presence (or the absence) of a quality, as in is light, has wings, or can fly. Semantic traits are unary predicates. A priori, there are no constraints on the distribution of traits across the various objects. Any combination may potentially be observed. On the other hand, the structure of the attributes is formally more complex and constrained. An attribute is composed of a set of exclusive values or modalities, which makes up its domain. For instance, the shape attribute may have different values, such as square, circle, or triangle. The coloured attribute may be instantiated as red, blue, etc. Unlike traits, the values of attributes are formally bound to each other: there is a structural link between red and blue which does not exist between red and square. Later, we will interpret this link in terms of the semantic distance between properties.

\section{Generalising the attribute structure}

We suggest that the trait structure and the attribute structure should be seen as particular cases of a more general structure featuring variable depth. Let us take the example of colours. The coloured property is a trait as some objects are coloured while others are not. We may prefer to be more specific; in which case, coloured becomes an attribute whose values will be red, blue, and so on. We may need to be even more precise, and, for instance, specify a particular shade of red. At this point, red becomes an attribute, whose values are vermilion, carmine, and so on. This implies that the same semantic content may be viewed simultaneously as an attribute or as the value of an attribute, depending on the degree of specificity that has to be stated. Thus, red is a value as regards the coloured attribute, but an attribute as regards the vermilion value. 
This analysis can be used for all kinds of properties. For structural properties, we may have, for instance, to have parts allowing movement, to have wheels, to have car wheels, to have racing car wheels, and to have Ferrari racing car wheels. The functional properties have the same hierarchical structure. From a general point of view, an object can be seen as movable. But to be more specific, one can say that the object can move by itself, that it moves on the ground, that it uses its legs to do so, that it runs, and so on.

Taking into account properties at different levels of generality-specificity leads us to consider them as being organised in hierarchical property lines. For each line, properties are linked to each other by means of an implication relation. Thus, we have [vermilion $\rightarrow$ red] and [red $\rightarrow$ coloured], where $\rightarrow$ means implies. The above line can also be expressed as follows: [/coloured/ red/vermilion]. This hierarchical property structure reflects the embedding of corresponding classes, as coloured objects include red objects, and red objects include vermilion objects.

From a psychological point of view, our conception of properties is compatible with the proposition set forward by Rosch et al. (1976) regarding a basic level in categorical structures. Using the property lines approach, we propose to look at the possibility of a correspondence between the basic level of categories and a preferred level in the hierarchical encoding of properties. This basic level for properties may correspond to the modality level of usual attributes like colour or shape, the superordinate level then being the level of the attribute as such. For example, in the [/coloured/red/vermilion] line, red will be at the basic level and coloured at the superordinate level. On the other hand, we have to consider that fruit (superordinate level) is coloured, while bananas (basic level) are yellow. The same analysis applies to structural and functional properties: to have car wheels (basic level) vs to have parts allowing movement (superordinate) or, in another example, to run (basic level) vs to move (superordinate). Tversky and Hemenway (1984) showed that the main properties that appear at the basic level are parts of the object, which corresponds to the idea that this level specifies how the function is realised.

\section{The property tree}

Generalising the notion of an attribute leads us to consider variable depth property lines with a priori implicative links between successive levels, from subordinate to superordinate ones. A priori means that, at this point, we do not need to take into account the way in which properties are distributed across different objects in order to identify those implications. To complete our hypothesis about property lines, we should say that lines are not to be seen as independent from each other, but as organised into property trees. For this reason, in addition to intra-line links (implications), we must consider transversal links related to line intersections. 
Line intersections are obviously needed if we want to express the above standard attribute-value structure using property lines. In actual fact, any attribute may be drawn as a two-level property tree, with one level for the value and one for the attribute. This tree can be directly interpreted in terms of property lines of [/attribute/value] form, with one line per value. We can see that the attribute level is the intersection point of all those lines. For example, consider the tree formed by the colour attribute and a set of values like red, blue, and so on. Two kinds of a priori links between properties can be found in this tree: firstly, intra-line links reflecting that, for instance, if an object is red, it is necessarily coloured; secondly, inter-line links which relate the various modalities of a given attribute. In the case of the colour example, these inter-line links show that when we say that two objects are of a different colour, we implicitly state that these objects look alike, in so far as our statement reflects the fact that they are both coloured.

Let us now consider an important topic that will help in the construction of property lines: the root of the property tree. The root of the property tree corresponds to an empty property, that is to say a property devoid of semantic content. As we move up the property tree, following a property line from subordinate levels to superordinate ones, the amount of semantic content we encounter will gradually decrease. Consider, for instance, the [/coloured/red/vermilion] line. When moving from vermilion to red, we lose the specificity of the red. When moving from red to coloured, we lose the specificity of the colour. As coloured is the line's top-level property, moving from coloured to the empty property causes us to lose the entire semantic content. It follows that any pair of lines forms a property tree, even if they belong to disjunctive semantic fields, in so far as they at least share a root node that means is a property. Consequently, a set of semantically independent traits may be seen as a one-level property tree.

Generalising the attribute structure enables us to join, within a single tree structure, any set of property lines we need to take into account when describing an object. Let us consider, for example, the three attributes colour, shape, and size. Despite the fact that each of them has its own set of modalities, we can look at those attributes as being three values of the appearance super-attribute. On the other hand, the rounded modality of the shape attribute can as such be seen as an attribute, as regards its egg-shaped value. The global tree of Figure 1 now starts to emerge.

\section{Describing objects using property trees}

A property tree like the one in Figure 1 represents a domain of description, a frame within which the actual description of objects will take place. The description of a particular object will be a sub-tree, a projection onto the entire domain, every line in the description starting at the empty property 


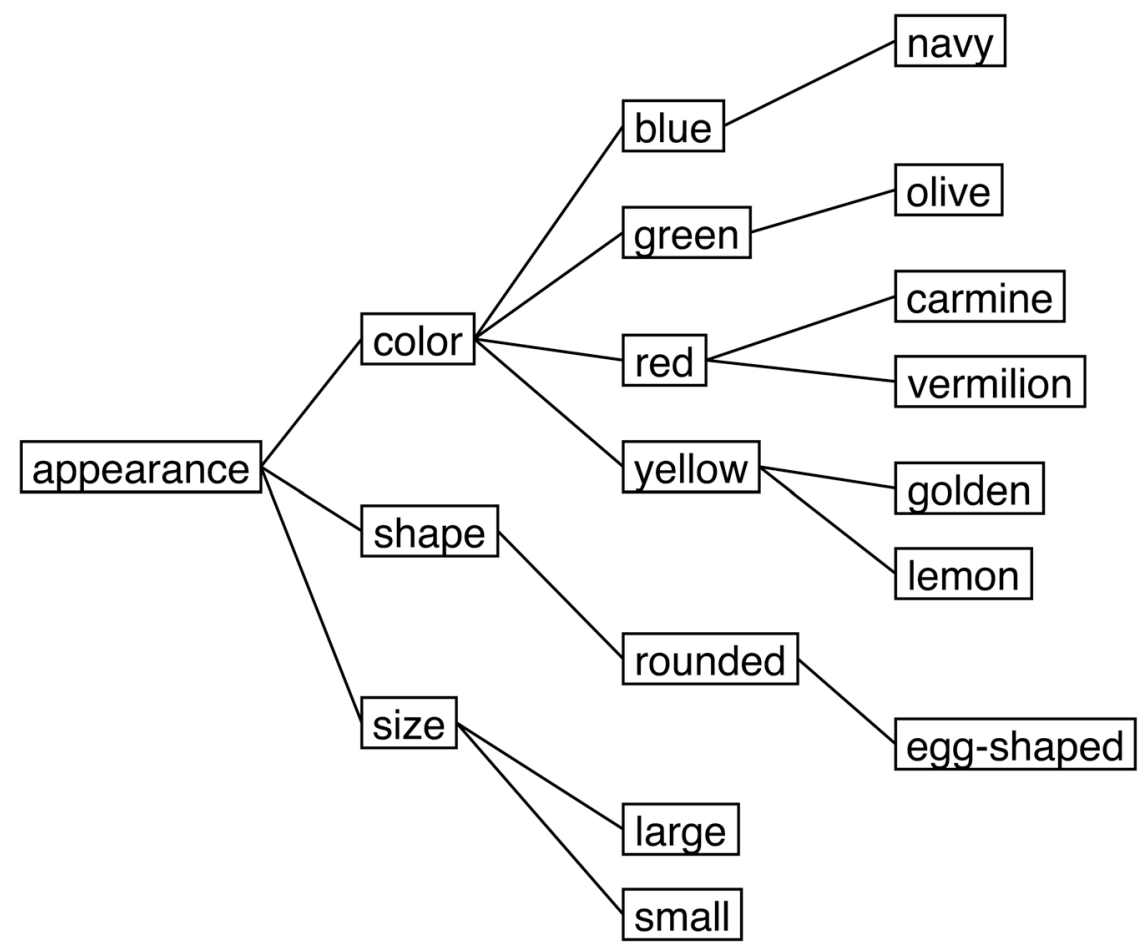

Figure 1. Generalisation of the attribute structure: (i) coloured, shape, and size attributes may be seen as values for the super-attribute appearance; (ii) the rounded value of the size attribute may be seen as an attribute regarding the egg-shaped value.

root. We will call this kind of domain tree subset the descriptor of an object. Figure 2 shows the descriptor of a small rounded lemon-yellow object, projected onto the domain tree shown in Figure 1.

It is important to notice that, in a property tree describing a single object (the descriptor of that object), modalities are not necessarily exclusive. Most objects have a shape and a colour. But some have a shape but no colour: they are translucent. Some have a colour but no shape, like smoke or mist. Finally, some objects have neither shape nor colour: they are impalpable and invisible. This is true at any level in the tree. Thus, in the example in Figure 2, the object described by the black nodes happens to have only one colour, unlike the rennet apple, which is multicoloured.

\section{Examples of property trees}

We may wonder whether the formal notion of property tree may be extended beyond the field of the physical appearance features such as form, 


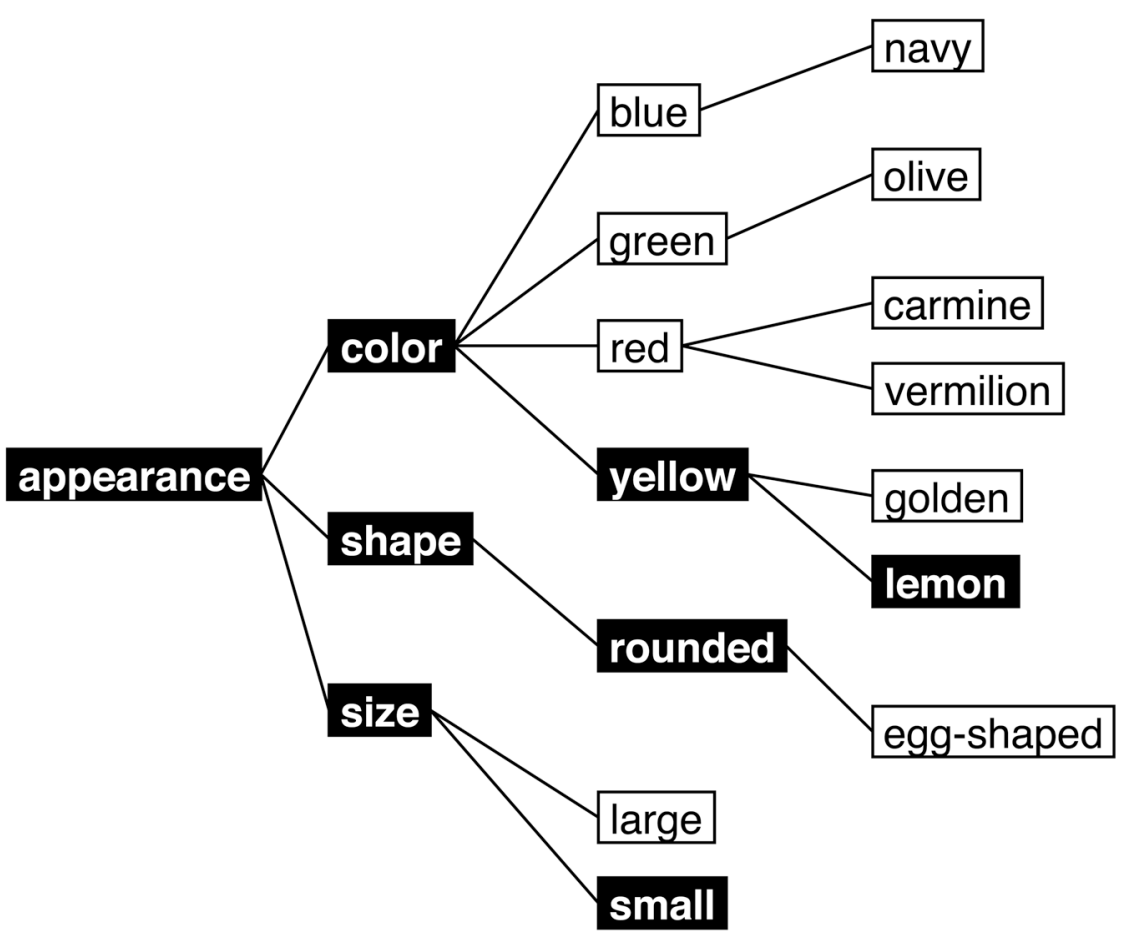

Figure 2. Black nodes form the descriptor tree of a particular object as a projection onto a larger tree that constitutes the domain of description.

size, and colour, and applied to complex domains characteristic of common natural categories such as animals, plants, foods, artefacts ...

In their description of the meaning of mental verbs, Rips and Conrad (1989) did indeed give an example of what we call a property tree, although their results were not interpreted in this way. They reported data showing that "one mental activity is a part of another if the second is a kind of the first" (p. 187). For instance, if reasoning is a kind of thinking, then thinking is part of reasoning. They interpreted their results as showing a difference between the categorisation of verbs and the categorisation of nouns, which raised a dispute (Fellbaum \& Miller, 1990; Rips \& Conrad, 1989, 1990) In their comments, we find it surprising that neither Rips and Conrad (1989, 1990), nor Fellbaum and Miller (1990) ever foresaw the following interpretation, namely, if reasoning is a kind of thinking, then the meaning of thinking is a part of the meaning of reasoning, as a result of the duality of the extensional vs intensional point of view of concepts. In the framework of the property-line approach, the semantic implication [reasoning $\rightarrow$ thinking] may be rewritten as [/thinking/reasoning]. In the latter property line, 
reasoning appears as a specification of thinking, thus the embedding of the two semantic contents is indeed a part-all relation, but one that operates at the level of the intension of concepts. The same phenomenon will be observed with any pair of properties linked by a semantic implication, as in red is a kind of colour vs the meaning of colour is part of the meaning of red.

Other results showing a hierarchical organisation of properties come from Ross and Murphy (1999) who used a food item sorting task and derived a similarity matrix from the sorting. They divided this matrix into matrices having a Robinson form according to the method developed by Hubert and Arabie (1994). In a matrix having a Robinson form, the values of cell entries decrease (or at least are equal) when one moves away from the diagonal along a row or a column. In such a matrix lines and columns are ordered according to a criterion, which is one-dimensional. In the primary dimension matrix obtained for two groups with one instructed to divide food items into groups "of similar food types" (taxonomic group) and the other into groups "of things that go together", there was a set of clusters strongly connected: in the first part are fruits, vegetables, cereals, and bread, in the last part dairy products, meat, and fish, and in the middle breakfast foods, snack foods (potato chips, pizza), and sweets (cake, cookies). As the authors propose, the ordering suggests a division between plant-based foods and animal-based foods. We may add that these supercategories regroup foods that are elementary or basic in that they have a single origin, while foods in the middle of the matrix are artefacts composed of several basic products. If we judge from the results obtained by Héron-Benaïcha (1998), fruits, vegetables, and cereals could be described by more general properties: the way they grow, what is eaten (leaves or flesh for vegetables, flesh but not seeds for fruits, grains [seeds] for cereals), whether there are seeds or not inside, etc. The supercategories of plant-based foods, animal-based foods, and handy-prepared foods are all related to the origin of foods, which constitutes a still more general property that can be contrasted with what Ross and Murphy call the script qualities of the foods. These are represented in the secondary Robinson matrix and regroup properties describing the circumstances of eating (the moment, the type of preparation). These two main divisions explain the quasi-totality of the variance of the data: they may be interpreted, as the authors suggest, as intrinsic properties versus properties describing how the things are used. Perhaps such extended organisation is characteristic of complex domains like food where a large amount of expertise is developed and the fact that such complex domains have been rarely investigated may explain why it has not been demonstrated until now.

To illustrate more precisely the methodology, which can be developed using the property line approach, we shall present the property tree that was behind the questionnaire along with some results obtained from this 
analysis. The property tree in Figure 3 is constructed with the data from the properties attributed in the judgement task for each of the 30 subcategories of fruits and vegetables by all the 10 participants, i.e., the properties about which there is a total agreement. Some properties present in the questionnaire are not represented in the graph ("season when it is harvested" for example), because there was not total agreement to assign this property to at least one of the 30 subcategories.

The property lines, which are the main branches of the tree are: type of, has appearance, grows, is made of, is eaten. For the property tree to be complete, it would be necessary to add a general property such as, is a food, which would be the root of the tree. The type of food is represented on the tree but it is not detailed, although it would be possible to define subcategories: for instance, among the fruits we may distinguish "citrus", "red berries", "dried", and among the vegetables "green", "starchy" ... The reason is that fruits and vegetables are categories and as such they are properties in a very specific way, in that they may be used to describe objects, but they are not basic properties like has a [/colour/blue/navy blue].

While basic properties may be represented as property trees, categories cannot: the network of categories usually has the form of a lattice, not of a tree. The property line [/is eaten] is the most complex, as we may expect. The first level of decomposition of this property is made of different points of view about the way a thing is eaten: how it is eaten (type of preparation), what is eaten (entirely or by removing something; as a matter of fact the node what has been collapsed with the node by removing something, because in the data there was no item recognised by all participants as being eaten in its entirety). The numbers present at the nodes are frequencies, i.e., the number of items (over 30) judged as having the property. At higher-level nodes the frequencies are cumulated: the frequency appearing at node [/grows/on something] is 10 , which means that besides the three participants who responded "grows on a tree" and the participant who responded "grows on a bush", there were six participants who responded "grows on something". For nodes where there was no question in the questionnaire (i.e., has a form), the frequency is the exact sum of the frequencies at the lower level.

Consider now the description of two fruits, bananas and peaches (Figure 4). The graph immediately allows us to see what is common and what is specific for both. In the usual descriptions a large part of what is common is left implicit, because it is implied by what is given at the more specific levels. Unfortunately this leads to a major underestimation of what is common as compared to what is specific.

Much remains to be done in order to have a complete property tree for a domain like food. However there is substantial agreement between the results of Héron-Bénaïcha (1998) and those of Ross and Murphy (1999), in 


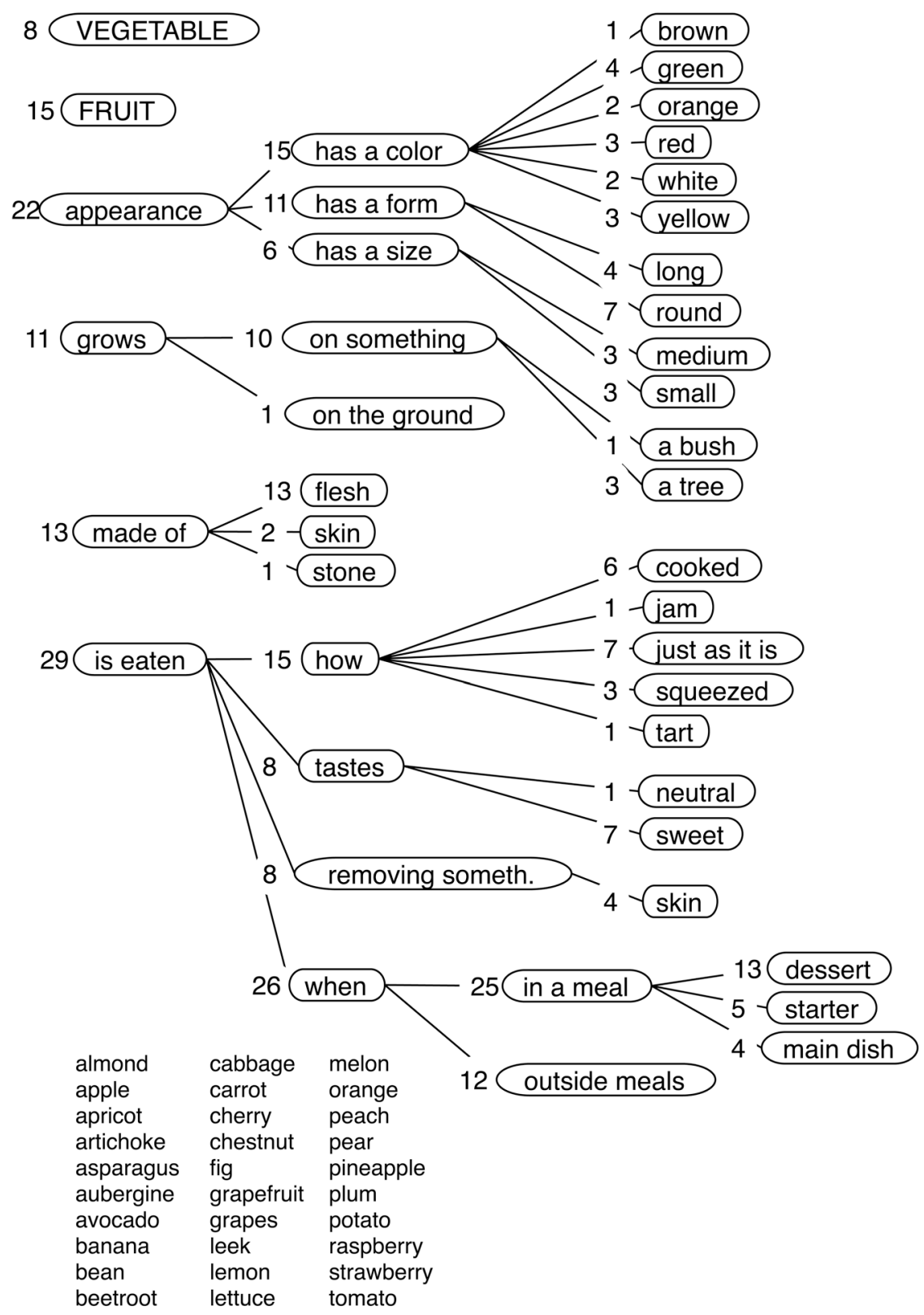

Figure 3. Property lines for fruits and vegetables according to Héron-Bénaïcha's data (see text for explanation). In front of each node is the number of subcategories (listed at the bottom of the figure) for which there is a total agreement between participants for the attribution of the corresponding property. 

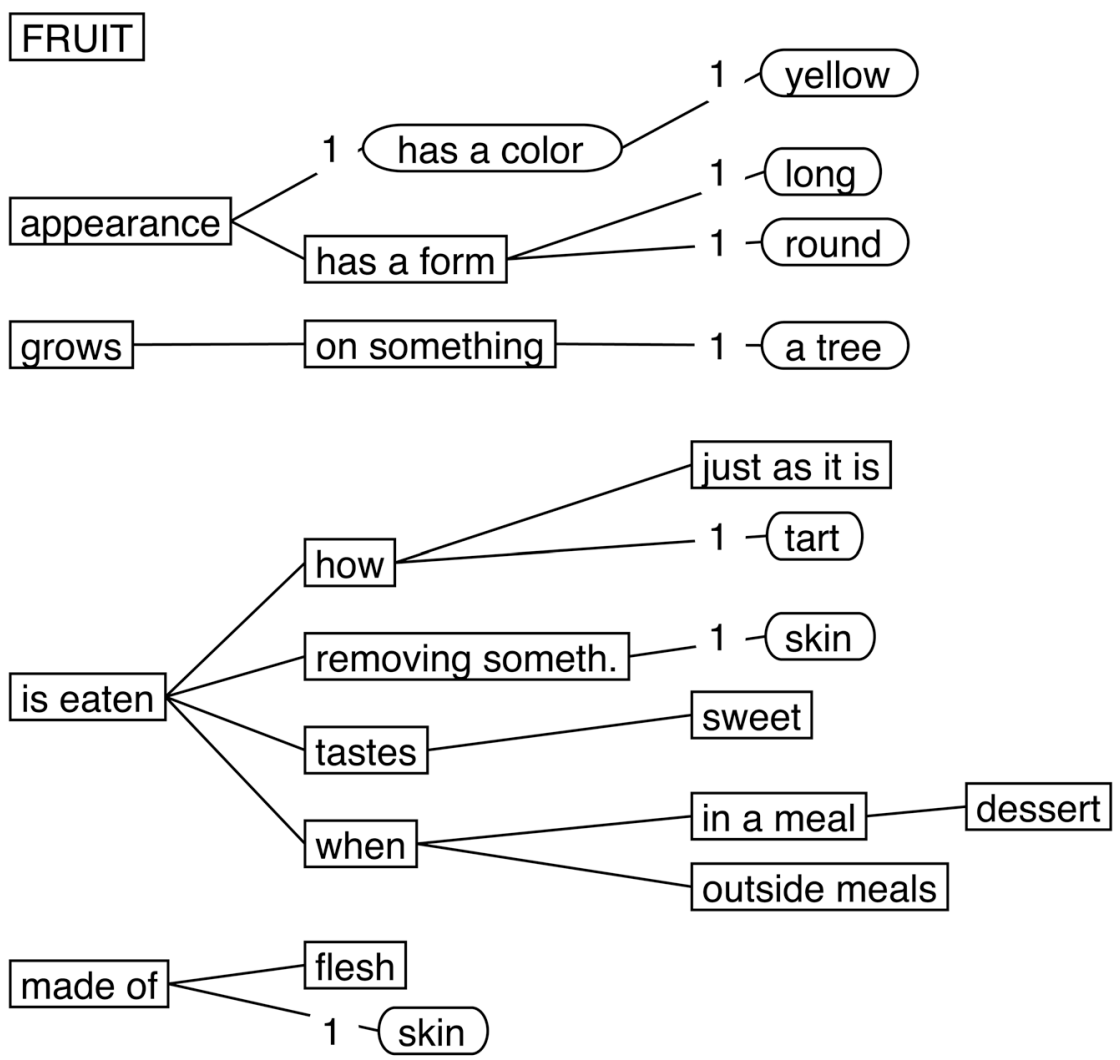

Figure 4. Description of two fruits, bananas and peaches. The nodes represented with rectangles correspond to the properties that are common to both fruits, and those represented with ellipses correspond to the differences. The graphs reads: concerning appearance, one has a colour that is yellow, both have a form, one long, the other round, etc.

spite of the differences in the tasks. Clearly there is much more similarity between a judgement task and a sorting task than there is between a judgement task and a property generation task. We attribute this to the fact that the context is very large in a sorting task ( 45 items in the study by Ross and Murphy) as compared with an isolated item in the property generation task.

We now want to show what type of results can be obtained using the methodology we propose by presenting the property tree obtained by Chauveix and Egoroff (2003) in a domain that is restricted but still complex - the domain of evaluating the texture of fabric. The elaboration of the property tree consists of a series of steps: sorting the fabric samples, verbalisation of the properties of each class by each participant, group 
discussion to reach a consensus concerning a list of properties, and regrouping the properties that seem similar until no more regrouping is felt possible. To validate the tree, if two properties $a$ and $b$ are in the same property line and if $b$ is more specific than $a$, then (i) the presence of $b$ has to be recognised as implying the presence of $a$ and (ii) each time $b$ is assessed for an item, $a$ has to be assessed for the same item. As the tree is big, we shall present only the roots of the main branches of the property tree and the details of a branch (namely rough). When two terms are equivalent, the second is parenthesised. The more general properties are: stiff, rough, smooth (supple), soft, unsoftened. The sub-tree for the rough property is shown in Figure 5.

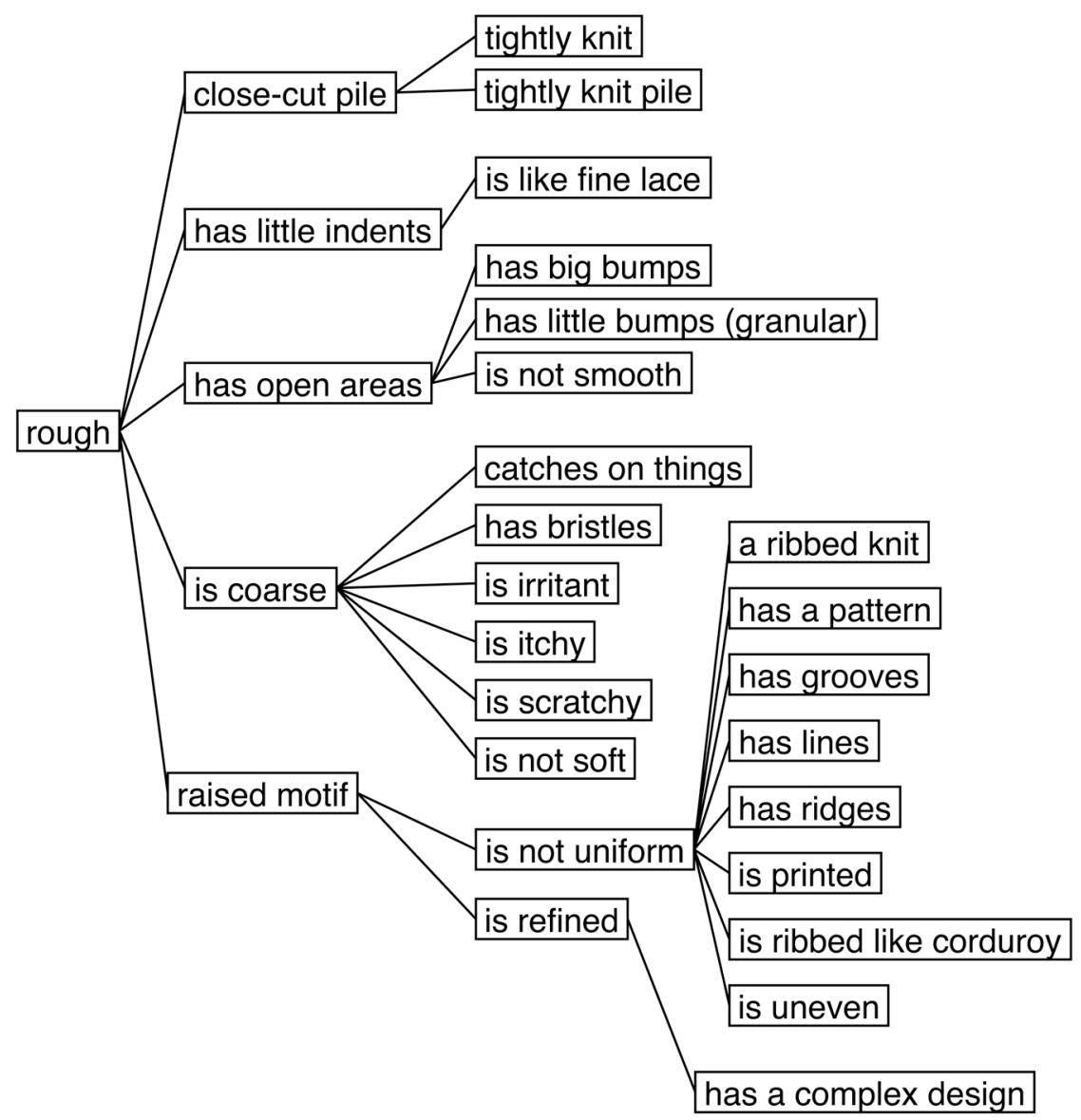

Figure 5. Sub-tree for the rough property. 


\section{A THEORY OF THE STRUCTURE OF NATURAL CATEGORIES AND OF CATEGORISATION IN CONTEXT}

Any theory of categorisation has to explain two types of phenomena: (i) the differences in accessibility of the various exemplars of a category from the label of the category, which are related to the typicality effects; and (ii) the differences in accessibility of the properties belonging to an object as a function of the context constituted by the set of objects that is presented together with it. The first aspect concerns the structure of categories in long-term memory and the type of access is top-down oriented, since the search for exemplars is based on the cognitive content of the category. The second one concerns the specific process of categorisation (corresponding to the notion of ad hoc categorisation proposed by Barsalou, 1983), which always takes place in a given context with a given situation and a given goal.

\section{The structure of natural categories}

Class inclusion is a major characteristic of natural categories as well as of formal concepts. The Galois lattice formalises how sets of objects are related to sets of properties, in embedded formal concepts.

The Galois lattice of a binary relation (Barbut \& Monjardet, 1970) is a hierarchical algebraic structure that has been used to investigate categorisation problems (Bernard \& Poitrenaud, 1999; Guénoche \& Van Mechelen, 1993; Poitrenaud 1995; Storms, Van Mechelen, \& De Boeck, 1994). Given a set of objects and a set of properties, we can build a binary relation composed of the set of all the object-property couples. This binary relation may be presented in a two-way table, like the one in Table 1, with properties in lines and objects in columns, where the boxes corresponding to the object-property pairs are ticked. If, for example, we look at the way some of the basic structural properties of geometrical shapes are organised, we

TABLE 1

Binary relationships between three basic properties and four geometrical objects

\begin{tabular}{lcccc}
\hline & A square & $\begin{array}{c}\text { A typical } \\
\text { rectangle }\end{array}$ & $\begin{array}{c}\text { A typical } \\
\text { rhombus }\end{array}$ & $\begin{array}{c}\text { A typical } \\
\text { parallelogram }\end{array}$ \\
\hline $\begin{array}{l}\text { Parall. opposite sides } \\
\text { Right angles }\end{array}$ & $\mathrm{X}$ & $\mathrm{X}$ & $\mathrm{X}$ & $\mathrm{X}$ \\
Equal sides & $\mathrm{X}$ & $\mathrm{X}$ & & \\
\hline
\end{tabular}


first obtain the binary relation set out in Table 1, then compute the corresponding Galois lattice, shown in Figure 6 in the form of a Hasse diagram.

The graph in Figure 6 shows how the Galois lattice is used to formalise the class inclusion relation underlying the data in Table 1 by correlating: (i) the set of objects and (ii) the set of properties. The resulting structure is a two-way lattice in which, when a set of objects A includes a set of objects B, the set of object properties for $\mathrm{A}$ is included in the set of object properties for $\mathrm{B}$, and vice versa. The presentation of this lattice in Figure 6 is not cumulative, as each object and each property appears only once although they can be inherited by several nodes, i.e., deduced by transitivity according to the two opposite order relations.

Wille (1984) calls the nodes of this graph formal concepts, because they clearly express the classic duality between concept extension and intension. Indeed, each node in Figure 6 corresponds to: (i) the set of all objects

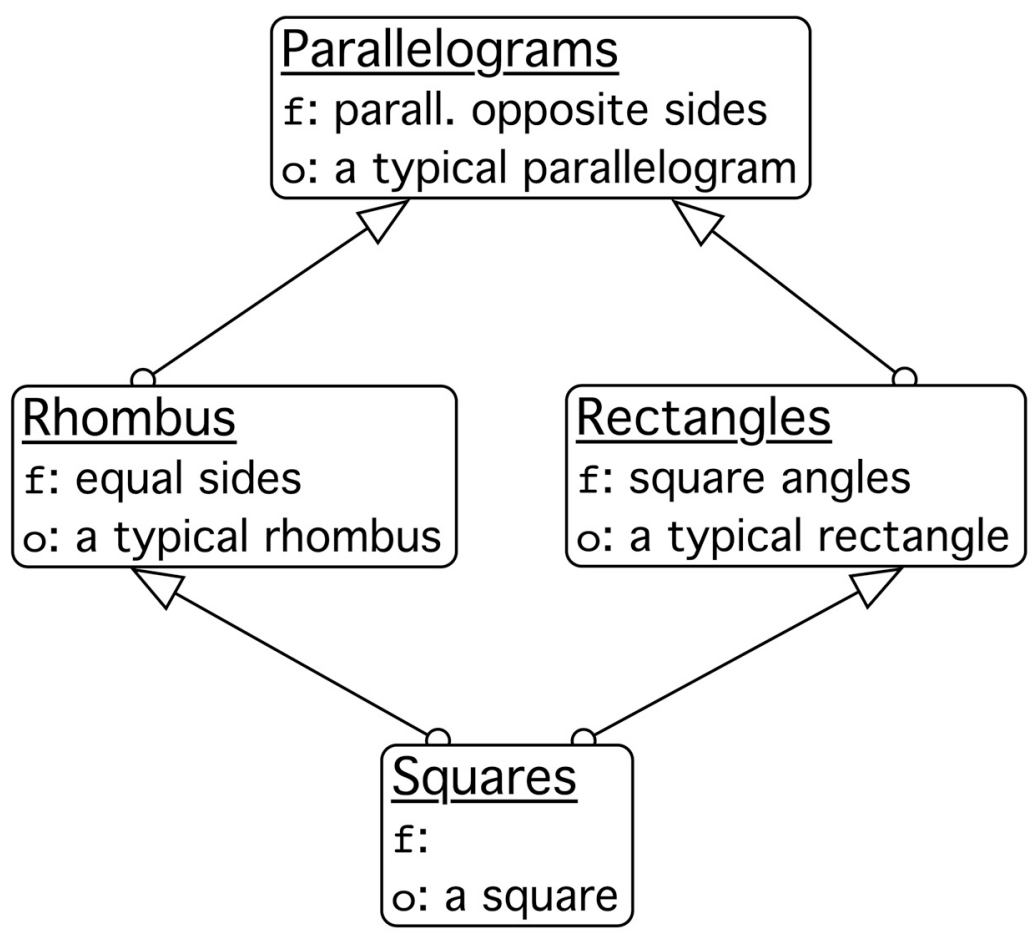

Figure 6. The Galois lattice resulting from the computation of the binary relations in Table 1. Each node refers to (i) a set of features and (ii) a set of objects. The underlined label is the name of the corresponding category. 
sharing a given list of properties, thus forming the extension of the concept; (ii) the set of all properties shared by those objects, thus forming the intension of the concept. For instance, the set of objects a typical rectangle, a square and the set of properties \{parallel opposite sides, right angles\} correspond to the concept of Rectangles as defined by mathematicians. In the extension of this we find those rectangles that are not squares (typical rectangles) as well as those that are. Following the arcs of the graph in the direction of the arrows, moving from specific concepts to general ones, we can directly read the implications underlying the hierarchical structure of the lattice: [right angles $\rightarrow$ parallel opposite sides] and [equal sides $\rightarrow$ parallel opposite sides].

At first glance, the Galois lattice seems difficult to use as a modelling tool for natural categories because the resulting structure is far too complex. It is indeed the case that if we take into account the precise way in which properties are distributed across the various objects, we end up with a highly

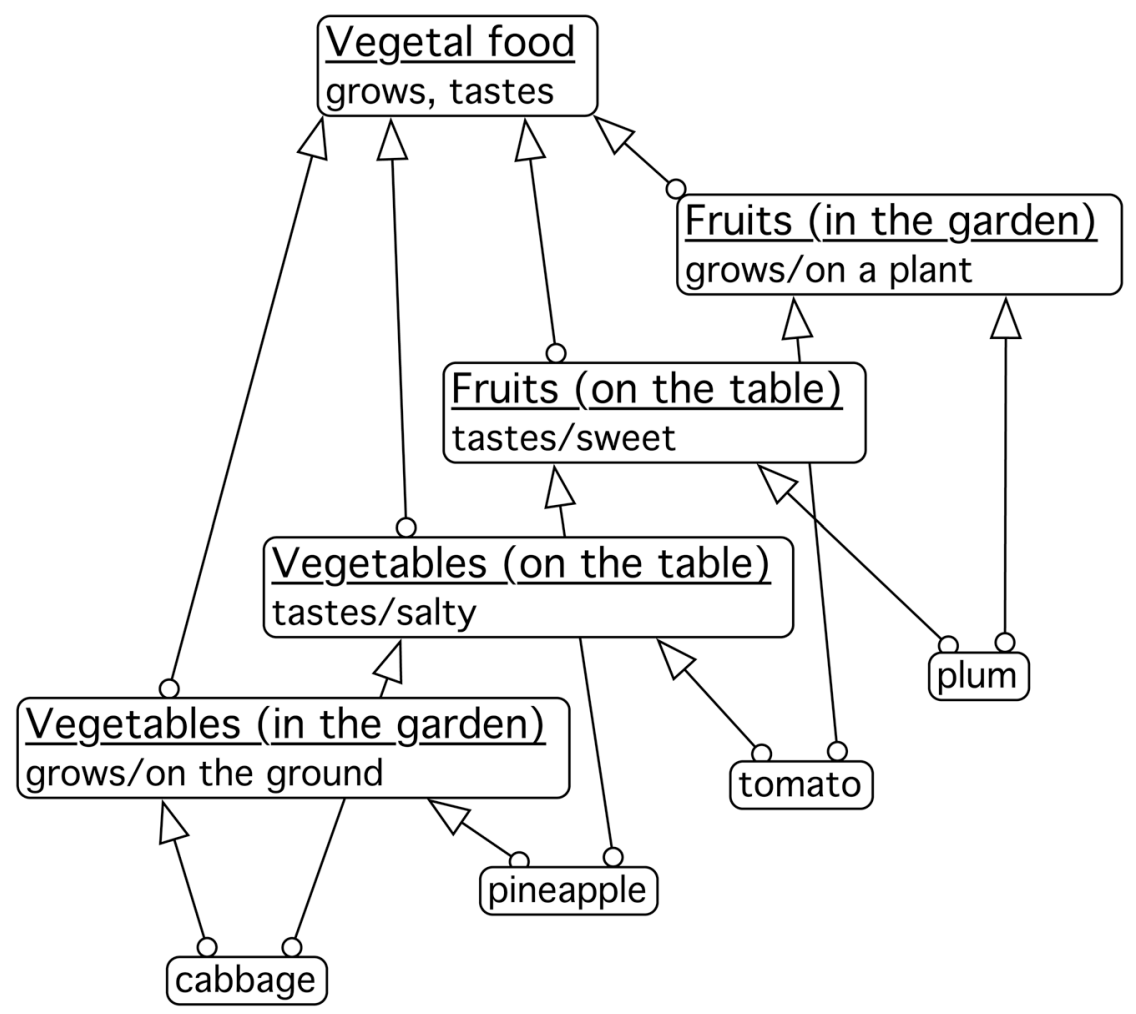

Figure 7. Four unordered categories of vegetal food have been generated in the lattice, reflecting the way properties are distributed across objects. 
combinatory lattice that does not reflect the rather simple organisation of natural categories. Figure 7 shows this clearly. Let us consider (i) some basic properties of fruits and vegetables, i.e., the way they grow (on the ground or on a plant) and the taste they have (sweet or salty); (ii) four subcategories of vegetal food, i.e., cabbage, pineapple, tomato, and plum. The resulting lattice reveals four formal concepts. Given the way the above properties combine, the natural categories of fruits and vegetables do not emerge as such, but are broken down in a non-hierarchical way. This is due to the fact that we consider (i) tomatoes to be fruits in the garden but vegetables on the table, and (ii) pineapples to be vegetables in the garden but fruits on the table. The culinary and biological points of view are conflicting, highlighting the polysemic nature of nouns for natural categories.

Obviously, the problem we are facing here has to be related to the typicality question. We suggest that tomatoes and pineapples disrupt the lattice because they are exceptions: they are both atypical fruits and atypical vegetables. Most fruits taste sweet. Most vegetables grow on the ground. This is why, on the other hand, the plum is a typical fruit and the cabbage a typical vegetable. If we now weigh the objects in the lattice in order to reflect the frequency of the properties in the subcategories of fruits and vegetables, (i.e., if we consider a typical vegetable and a typical fruit, each one standing for a list of such vegetables and fruits), the four properties will correlate as shown in Figure 8. From a given threshold, we will have what were previously called quasi-implications. In other words, if we set aside borderline cases like tomatoes and pineapples, [/tastes/salty] and [/grows /on the ground] on the one hand, and [/tastes/sweet] and [/grows/on a plant] on the other hand, correlate.

We have seen that in the Galois lattice, when two properties imply each other, and are thus logically equivalent, they share the same categorical node. The resulting structure (Figure 9) is far simpler than the previous one (Figure 8) because the four categories Vegetables (in the garden) and Vegetables (on the table) on one hand, and Fruits (on the table) and Fruits (in the garden) on the other hand, have merged into pairs. Furthermore, the new structure matches common knowledge about what is called a fruit or a vegetable.

The simplified lattice in Figure 9 leads to the following conclusion: the properties of a natural category are the properties of its typical exemplars. In this way, natural categories are simplifications, maximising correlations between properties by excluding cases for which the correlations do not hold well. Thus, we arrive at the same conclusion that the holders of the prototype view draw for prototypes. We differ as regards atypical cases. As we will see later, these borderline cases may, however, be referred to by the verbal labels of natural categories in an atypical way. In short, simplification results in an economy of verbal labels for categories as compared to the 


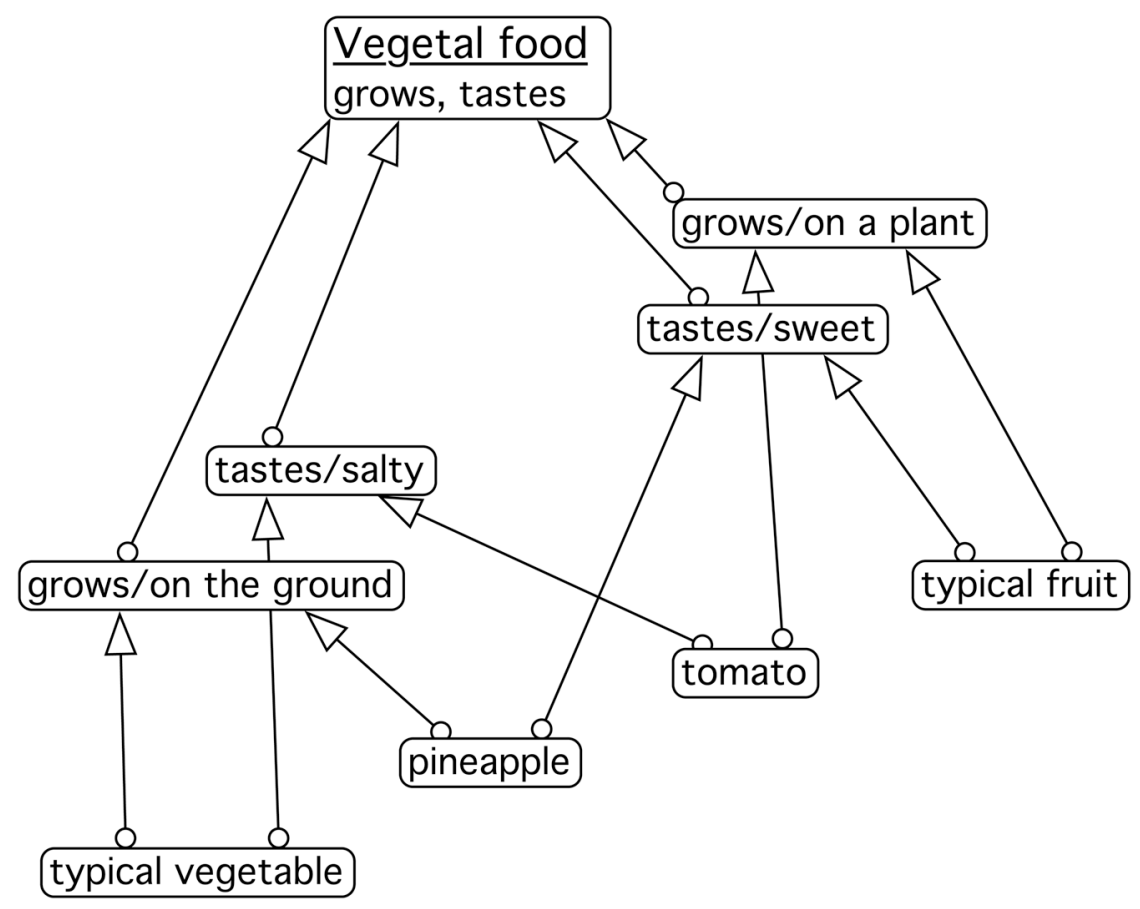

Figure 8. Typical entities have a greater weight than atypical ones, producing a mutual attraction in the graph between correlated properties.

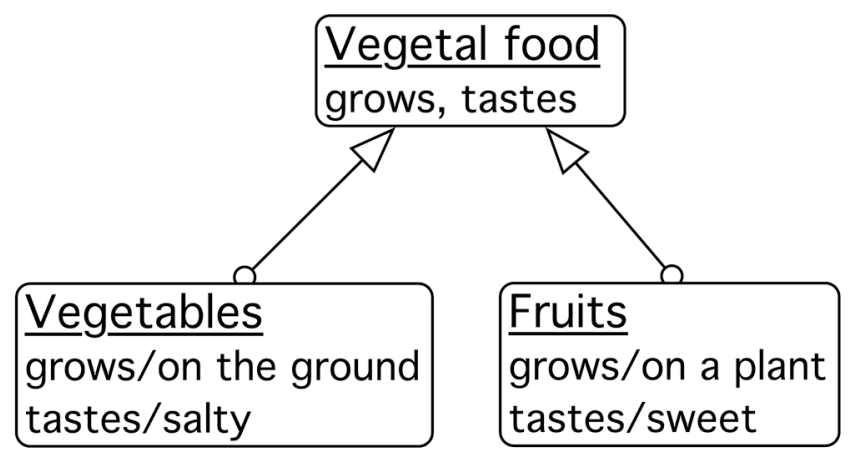

Figure 9. The simplified graph obtained when untypical exemplars are excluded on the grounds of the quasi-implication principle: The natural categories of Fruits and Vegetables are emerging.

richness of cognitive categories being referred to. Consequently, as we will explain later, the relation of verbal labels to categories is not one-to-one: the same label may refer to several different categories. 
Fruits grow on plants and we eat them as a sweet. Vegetables grow on the ground and we eat them in salty dishes. This can be called typical knowledge, as it is usually the case. However, in specific circumstances, things may appear somewhat differently. Indeed if we are in the garden, the accessibility of properties for fruits and vegetables will be different from that in the dining room. In the garden, the way things grow is a property that matters. In that context, tomatoes are clearly fruits and pineapples are vegetables. In the dining room, taste is what matters, not the way things grow. Tomatoes then become vegetables and pineapples become fruits. This can be easily modelled using two alternative Galois lattices (Figure 10), in which some properties are highlighted while others remain in the shadow.

From the above point of view, atypical exemplars such as tomatoes and pineapples can be alternatively treated as fruits or vegetables, depending on the context. In a way, they are both fruits and vegetables, in that they inherit
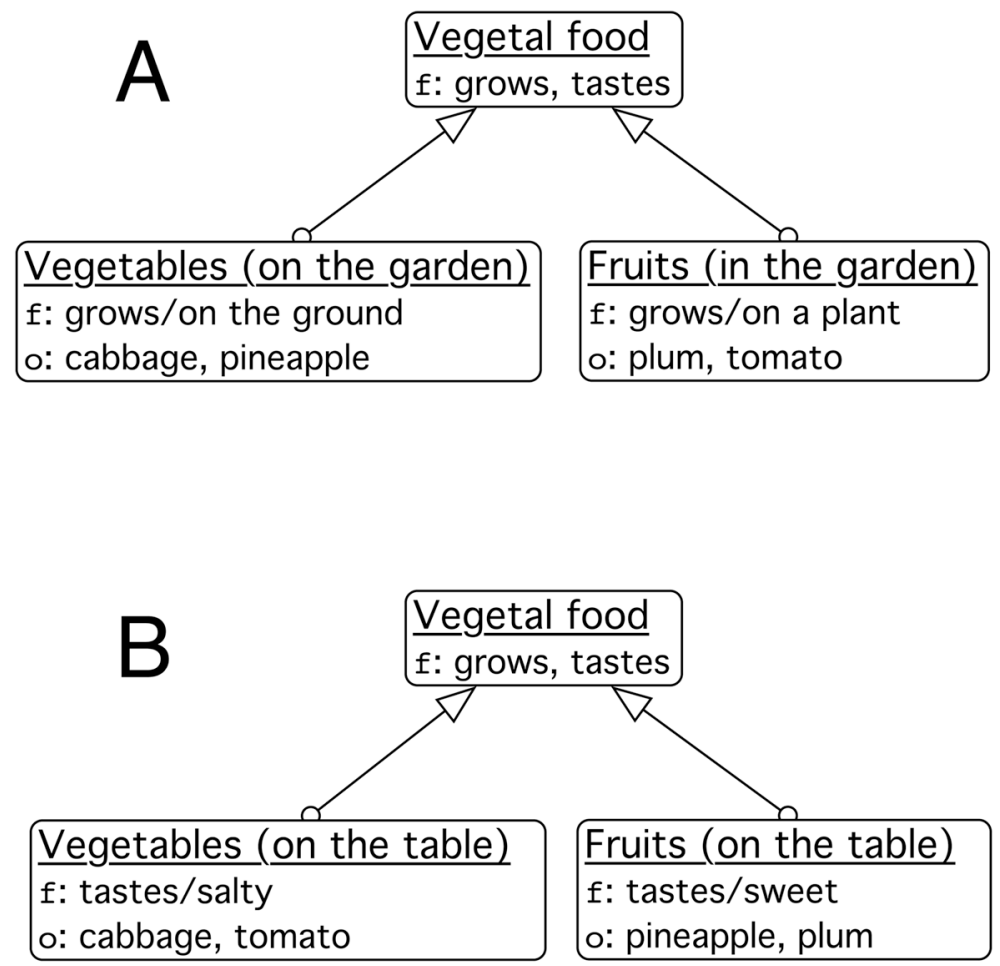

Figure 10. (A) The lattice associated with the garden context. (B) The lattice associated with the table context. In each case, the generic content of the shadowed property line (tastes for the garden and grows for the table) is still part of the representation and is a core property. 
properties from both sides, as shown in Figure 8. However, depending on the context, the variable component is the set of properties selected and made salient by the given context. The properties defining natural categories (the conceptual core), on the other hand, constitute long-term memory knowledge and, as such, generally remain stable. This is not the case for working categories that are created for the purposes of immediate goals in particular situations.

A number of questions are still open to debate regarding the notion of property lines. Is their list fixed? How do we investigate and describe the most abstract levels and also the most specific, those related with perception and mental images? It is obvious that the intermediate levels are easier to describe, because they are better conceptualised and usually expressed in language. A formalism does not solve these questions but it may help to have a new interpretation of existing data and provide some ideas for new experiments.

Let us apply this approach to the data of Héron-Bénaïcha (1998) presented previously. We can draw the Galois lattice showing how the properties, as well as the objects, are organised. We shall consider only the description of the 30 subcategories by the property lines when the item is eaten and how it tastes, and we shall add the property fruit or vegetable for the items that are recognised by the 10 participants as being a fruit or a vegetable

Let us show how the relations of the 30 items of food are related to the superordinate categories of fruits and vegetables using the Galois lattice approach. In the study by Héron-Bénaïcha (1998) participants were asked to name the categories to which each item belongs: food, drink, fruit, vegetable. All participants recognised 16 items as fruits and 8 as vegetables. The items that were assigned to neither of these categories were: apricot, almond, chestnut, tomato, avocado, asparagus, beetroot. Except for apricot (which was judged as a fruit by nine participants), these food items are rather atypical of fruits and vegetables. The Galois lattice was drawn for the 30 items with the categories of fruits and vegetables added. The result is presented in Figure 11 for a part of the property tree, i.e., the property line how it is eaten. A property is retained for an item if there is agreement for all 10 subjects.

Although the description is far from being complete (since only the properties for which there was a complete consensus were retained), this graph is very informative about the cognitive content of categories of fruits and vegetables. First the categories of fruits and vegetables are quite distinct. Fruits appear on the left of the graph and vegetables on the right. There is no overlap: there are properties that are characteristic of fruits, those that appear in node fruit, plus some that are more specific (such as [/is eaten/in a tart], [/is eaten/in jam], [/is eaten/squeezed]), and there are 


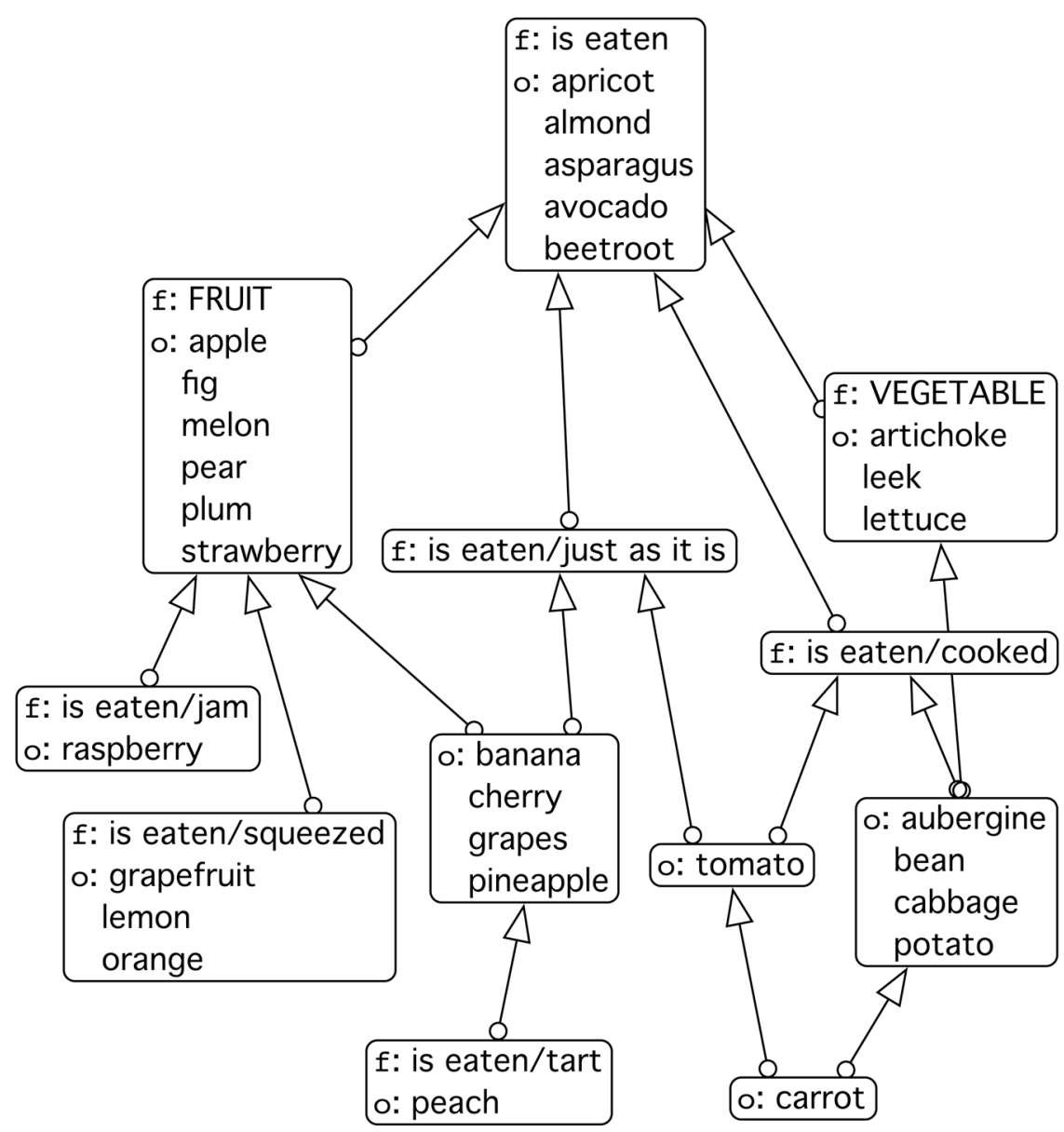

Figure 11. Galois lattice of the 30 food items (from Héron-Bénaïcha, 1998). Chestnut does not appear in the graph because there was no agreement between all participants about the way it is eaten.

properties characteristic of vegetables, plus a property common to almost all vegetables ([/is eaten/cooked]), and a property that belongs to a fairly large number of fruits and a few vegetables (tomato and carrot) appears in the centre of the graph, ([/is eaten/just as it is]). Clearly being cooked is not a characteristic property of the vegetables, since there are vegetables that are cooked to compose a dish, simply boiled or prepared with a dressing, or both. At the top are the items for which there is not a total consensus about whether they are fruits or vegetables. Tomatoes are also one item that is not recognised by everybody as a vegetable, but it has a supplementary property [/is eaten/cooked], common with most vegetables. We could make the same 
analysis with other property lines (for instance, when it is eaten, how it grows, how it tastes) and get the same results.

\section{Modelling the flexibility of categories}

The fact that the same object may be assigned to different categories as a function of the mental context of the moment (a tomato may be seen as a fruit in a garden context and a vegetable in a meal context) may be seen as a selective effect that can be modelled by using the Galois lattice approach combined with the property line approach. Consider a common description of the four food items we looked at before: cabbage, pineapple, plum, and tomato. The selective effect we consider is a focusing phenomenon generated by a high-level category: food versus garden vegetal items. This selective effect may be represented as the screening of the properties of the items considered using the properties of the high-level category. For example, in the case of the garden context the only properties that are retained are the

TABLE 2

Properties of four food items: cabbage, pineapple, plum and tomato

\begin{tabular}{|c|c|c|c|c|c|c|}
\hline & Garden item & Food item & Cabbage & Tomato & Pineapple & Plum \\
\hline Grows & $\mathrm{x}$ & & $\mathrm{x}$ & $\mathrm{x}$ & $\mathrm{x}$ & $\mathrm{x}$ \\
\hline on something & & & & $\mathrm{x}$ & & $\mathrm{x}$ \\
\hline a tree & & & & & & $\mathrm{x}$ \\
\hline a plant & & & & $\mathrm{x}$ & & \\
\hline on the ground & & & $\mathrm{x}$ & & $\mathrm{x}$ & \\
\hline Has a size & $\mathrm{x}$ & & $\mathrm{x}$ & $\mathrm{x}$ & $\mathrm{x}$ & $\mathrm{x}$ \\
\hline medium & & & $\mathrm{x}$ & & $\mathrm{x}$ & \\
\hline small & & & & $\mathrm{x}$ & & $\mathrm{x}$ \\
\hline Reproduces & $\mathrm{x}$ & & $\mathrm{x}$ & $\mathrm{x}$ & $\mathrm{x}$ & $\mathrm{x}$ \\
\hline has seeds & & & & $\mathrm{x}$ & & $\mathrm{x}$ \\
\hline stone & & & & & & $\mathrm{x}$ \\
\hline grains & & & & $\mathrm{x}$ & & \\
\hline Tastes & & $\mathrm{x}$ & $\mathrm{x}$ & $\mathrm{x}$ & $\mathrm{x}$ & $\mathrm{x}$ \\
\hline sweet & & & & & $\mathrm{x}$ & $\mathrm{x}$ \\
\hline neutral & & & $\mathrm{x}$ & $\mathrm{x}$ & & \\
\hline Is eaten & & $\mathrm{x}$ & $\mathrm{x}$ & $\mathrm{x}$ & $\mathrm{x}$ & $\mathrm{x}$ \\
\hline how & & $\mathrm{x}$ & $\mathrm{x}$ & $\mathrm{x}$ & $\mathrm{x}$ & $\mathrm{x}$ \\
\hline raw & & & & $\mathrm{x}$ & $\mathrm{x}$ & $\mathrm{x}$ \\
\hline cooked & & & $\mathrm{x}$ & $\mathrm{x}$ & & \\
\hline when & & $\mathrm{x}$ & $\mathrm{x}$ & $\mathrm{x}$ & $\mathrm{x}$ & $\mathrm{x}$ \\
\hline in a meal & & & $\mathrm{x}$ & $\mathrm{x}$ & $\mathrm{x}$ & $\mathrm{x}$ \\
\hline starter & & & & $\mathrm{x}$ & & \\
\hline main course & & & $\mathrm{x}$ & $\mathrm{x}$ & & \\
\hline dessert & & & & & $\mathrm{x}$ & $\mathrm{x}$ \\
\hline outside meals & & & & & & $\mathrm{x}$ \\
\hline
\end{tabular}


property lines belonging to the garden vegetal items, i.e., the properties of the garden vegetal items and those that are more specific. These properties are presented in Table 2 .

In the garden context the selection retains only the property lines [/grows], [/has a size] and [/reproduces]. So the properties retained are:

- for cabbage, [/grows/on the ground $]+[/$ has a size/medium $]+$ [/reproduces];

- for tomato, [/grows/on something/a plant] + [/has a size/small $]+$ [/reproduces/has seeds/grains];

- for pineapple, [/grows/on the ground $]+[/$ has a size/medium $]+$ [/reproduces];

- for plum, [/grows/on something/a tree + [/has a size/small $]+$ [/reproduces/has seeds/stone].

Similarly in the food context, the properties retained are:

- for cabbage, $[/$ tastes/neutral $]+[/$ is eaten/cooked $]+[/$ is eaten/in a meal];

- for tomato, $[/$ tastes/neutral $]+[/$ is eaten/raw $]+[/$ is eaten/cooked $]+$ [/is eaten/in a meal/starter] + [/is eaten/in a meal/main course];

- for pineapple, [/tastes/sweet $]+[/$ is eaten/raw $]+[/$ is eaten in a meal /dessert];

- for plum, [/tastes/sweet $]+[/$ is eaten/raw $]+[/$ is eaten/in a meal $/$ dessert $]+[/$ is eaten/outside meals $]$.

The Galois lattices corresponding to the descriptions given in each context are presented in Figure 12. Three categories emerge beside the four items, for example, in the garden context a general category corresponds to vegetal items with the properties [/grows], [/has a size] and [/reproduces].

Two intermediate categories regrouping the common properties to plum and tomato ([/grows/on something], [/small], [/has seeds]) and the properties common to cabbage and pineapple ([/grows/on the ground], [/has a size /medium]) emerge. As these categories have properties that are, as we have seen, characteristic of fruits and vegetables respectively, it appears that in the garden context plum and tomato are regarded as fruits, while pineapple and cabbage are seen as vegetables (at least for people who don't think that they grow on trees).

In the food context outside the general category of food (with properties [/is eaten/in a meal], [/is eaten/how], [/has a size], [/tastes]), two intermediate categories are produced similarly regrouping the properties common to plum and pineapple ([/tastes sweet], [/is eaten/raw], [/is eaten/in a meal/dessert]) and those common to tomato and cabbage ([/tastes/neutral], [/is eaten/cooked] 


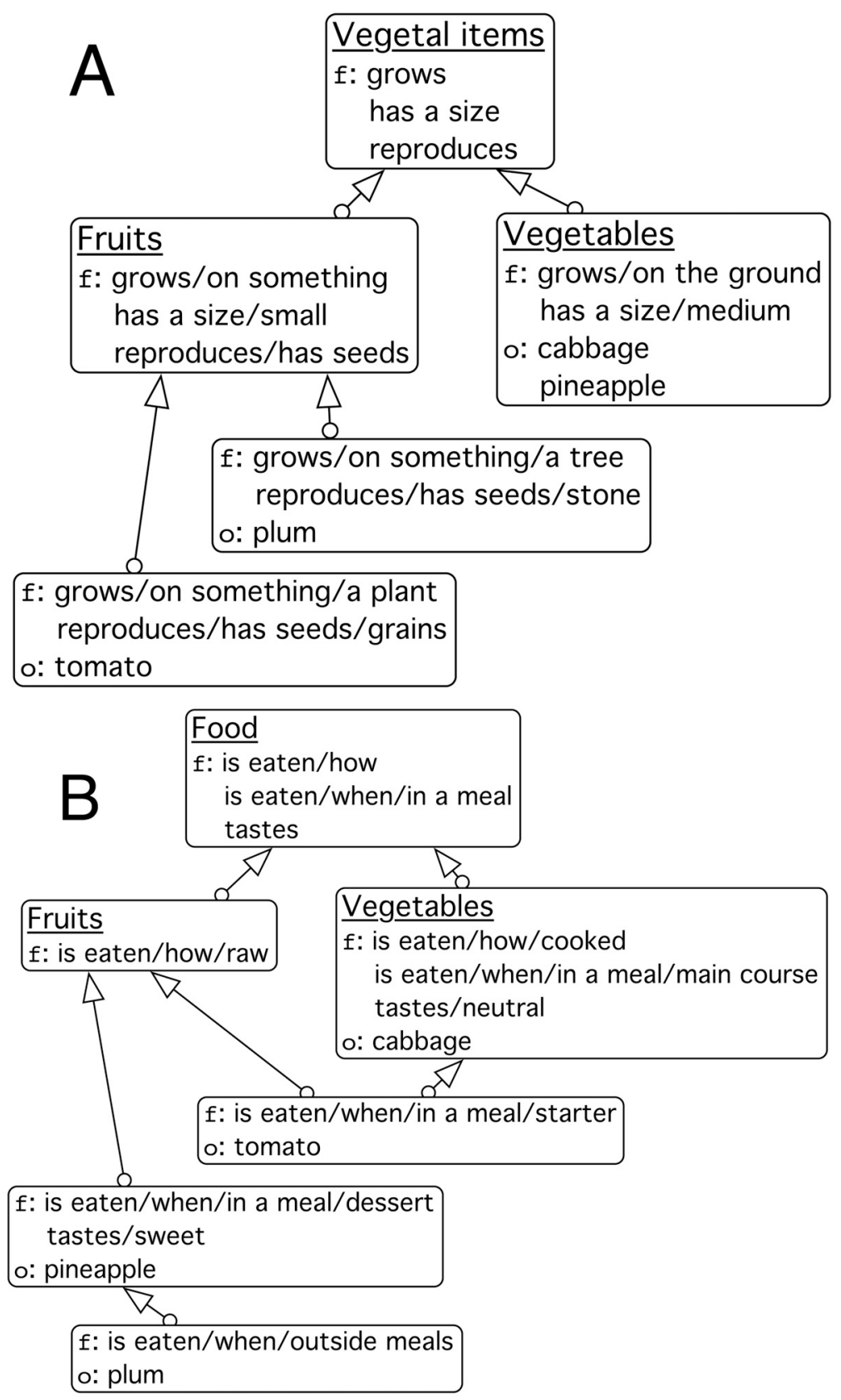

Figure 12. Gallois lattices for cabbage, tomato, pineapple, and plum: (A) the context of garden items, (B) the context of food items. 
and [/is eaten/in a meal/main course]). As these properties contrast fruit with vegetables, it follows that in a food context pineapple will be considered as a fruit together with plum, and tomato will be seen as vegetable like cabbage.

These examples of cross-classification are not due to a fuzzy definition of the concepts of fruits and vegetables but to the fact that different properties are selected for the items in context. In this way the same item may be referred to in two contrasting categories in different contexts. By the same process a verbal label may refer to different categories and therefore the flexibility of lexical labels must not be confused with fuzziness in category boundaries.

\section{The relation of labels to cognitive categories}

Cognitive categories must not to be mistaken for the noun used to refer to them. The relation between categories and names of categories is a complex one (see Gelman \& Heyman, 1999; Malt, Sloman, Gennari, Shi, \& Wang, 1999; Yamauchi \& Markman 2000). We do not want to discuss here the difference in information conveyed by a noun label and an adjective or a verb regarding the information conveyed nor the context effects, which can highlight some of the properties of the category and make them more salient-we shall insist on another phenomenon, which has also to be considered. This is related to the distinction between marked vs unmarked terms, which is familiar to linguistics (Greenberg, 1966). This distinction is obvious in the case of adjectives: we can say this rod is one foot long even if one foot is not "long" for a rod. Long is used to designate both the entire range of lengths and a specific range of lengths, those that are not short. The meaning of unmarked terms may be inclusive or contrastive, while the meaning of marked terms is necessarily contrastive. This phenomenon is also observed for nouns and Politzer (1991) claimed that it has major cognitive implications. For instance, the noun rectangle is interpreted inclusively in the sentence a square is a rectangle with equal sides but interpreted contrastively in the sentence $I$ s it a rectangle or a square? It may refer either to a class or to a sub-class. This happens when there is only one name for both the class and one of its sub-classes, although the polysemy of the term should not be taken as an ambiguity in the concepts, which are clearly defined. The mobility of lexical labels directly matches the stability of conceptual structures, according to the constraints imposed by the context.

It may even happen that a category name, which usually has only the inclusive meaning, is interpreted contrastively in specific contexts. Politzer (1991) argues that this is the case in the famous Piagetian experiment on class inclusion, featuring a set of two sorts of flowers (e.g., four daisies and two tulips). Here, the child is asked, "Are there more daisies or more flowers?" A 6-year-old child typically responds, "There are more daisies." 
This is usually interpreted as showing that the child has not built the class/ sub-class distinction. Politzer (1991), however, rejects this interpretation and claims that as the term flowers appears in the same sentence as daisies, it is interpreted in a contrastive way, as meaning flowers other than daisies. When, prior to the inclusion question, the child is asked "How many flowers are there?" then "How many daisies are there?", the majority of children aged 6 give a correct response to the inclusion question. However if the preliminary counts are made in the reverse order ("How many daisies are there?", followed by "How many flowers are there?") only about $20 \%$ of the children respond correctly. Clearly, when flowers appears first, it is interpreted in an inclusive way, whereas when it appears second, it is interpreted in a contrastive way. This effect can be observed for every type of label, whether verbal or pictorial.

The cognitive implications of this phenomenon have hardly begun to be drawn. We would claim that this is due to the fact that a property line may be considered at a more or less general level. Thus, the fact that unmarked terms may convey a more or less general meaning directly reflects a major property of cognitive processing: the ability to move along a generality/ specificity dimension. This principle is complementary to the principle of basic-level categorisation. There exists a favoured level of categorisation but it is possible to move from one level to another without any need for new labelling.

The failure of linguists to produce satisfactory definitions of the semantic content of words by stringing together primitives, (an argument raised by advocates of the prototype theory against the existence of a conceptual core), must be considered in this light. Given that the specific meaning of a word in a context depends both on its signification and on the context, it is a priori impossible to anticipate the whole set of these possible meanings and a fortiori impossible to give a definition encompassing all these possible meanings. A definition must be valid regardless of the context, while a natural category is always interpreted in a given context. It is necessary to distinguish between the conceptual content of the category and the interpretation that is conveyed by the name of the category in a given context. Interpretations are extremely variable and very broad. Phenomena such as emergent properties that arise from context effects such as concept combinations (Hampton, 1993) or metaphors (Tourangeau \& Rips, 1991) show that the list of possible interpretations of a word is open-ended.

\section{Categorisation in context}

We now have to examine the context effects, which are not semantic like those previously considered, but which arise from the physical context. The properties that are retained to describe an object in a given context are based 
on the nature of the other objects that are present in the same context. In this case it is a bottom-up selection effect, not a top-down one, as in the case of a mental context. This type of selection may be modelled in the same way using the Galois lattice mechanism and some additional assumptions. Consider a set of objects and a set of properties describing them, as well as a table showing which properties are present in each object. We assume that the properties that are retained as relevant are only those that are informative, namely those that are necessary and sufficient to show both the similarities and the differences; those that are common to the whole set and those that are specific to each item. More precisely, the basis for the selection of properties is the description of the set of objects given in a property lines format, which represents the information in long-term memory about each object.

A general category must be defined which will be the most specific possible and encompass all the objects of the set. The only property lines considered as being used in the description are those that are present in all objects of the set, and thus are relevant to the description

The property lines selected are specified using the perceptive (or semantic if the context is verbal) information present in the situation: at this step, the most general properties sufficient for defining what is specific to each object are retained. This aspect of description provides a description for each object in the context.

The Galois lattice of the table giving the description of all the objects of the context is computed and the result is a representation of the similarities and of the differences among the objects. The most common properties are at the top of the lattice and the most specific, in terms of distinguishing the objects from each other, are at the bottom. Let us consider the two examples of contexts presented in Figure $13 \mathrm{a}$ and b. Only one form changes from 13a to 13b: the black circle becomes white. This small difference produces an important change in the lattice.

The lattice extracts the categories that are common to several objects. At the top of the lattices, the more general have bidimensional geometric forms. In lattice a, the intermediate categories are square and black, while in lattice $\mathrm{b}$ they are square and white. The lattice makes obvious the properties that are specific to each figure. One form has no specific property, but only shared properties (form $\mathrm{M}$ in a and form $\mathrm{L}$ in $\mathrm{b}$ ). The two other forms have specific properties: white for form $\mathrm{L}$ and round for form $\mathrm{R}$ in a, black for form $\mathrm{M}$ and round for form $\mathrm{R}$ in $\mathrm{b}$. The description of form $\mathrm{M}$ changes from a to b: in order to identify this form we can say "the black one" in b, while it is necessary to say "the black square" in a. Nevertheless, the form has not changed, only its environment.

The Galois lattice captures an essential aspect of the context: the distribution of properties over objects. This is important, because it may 

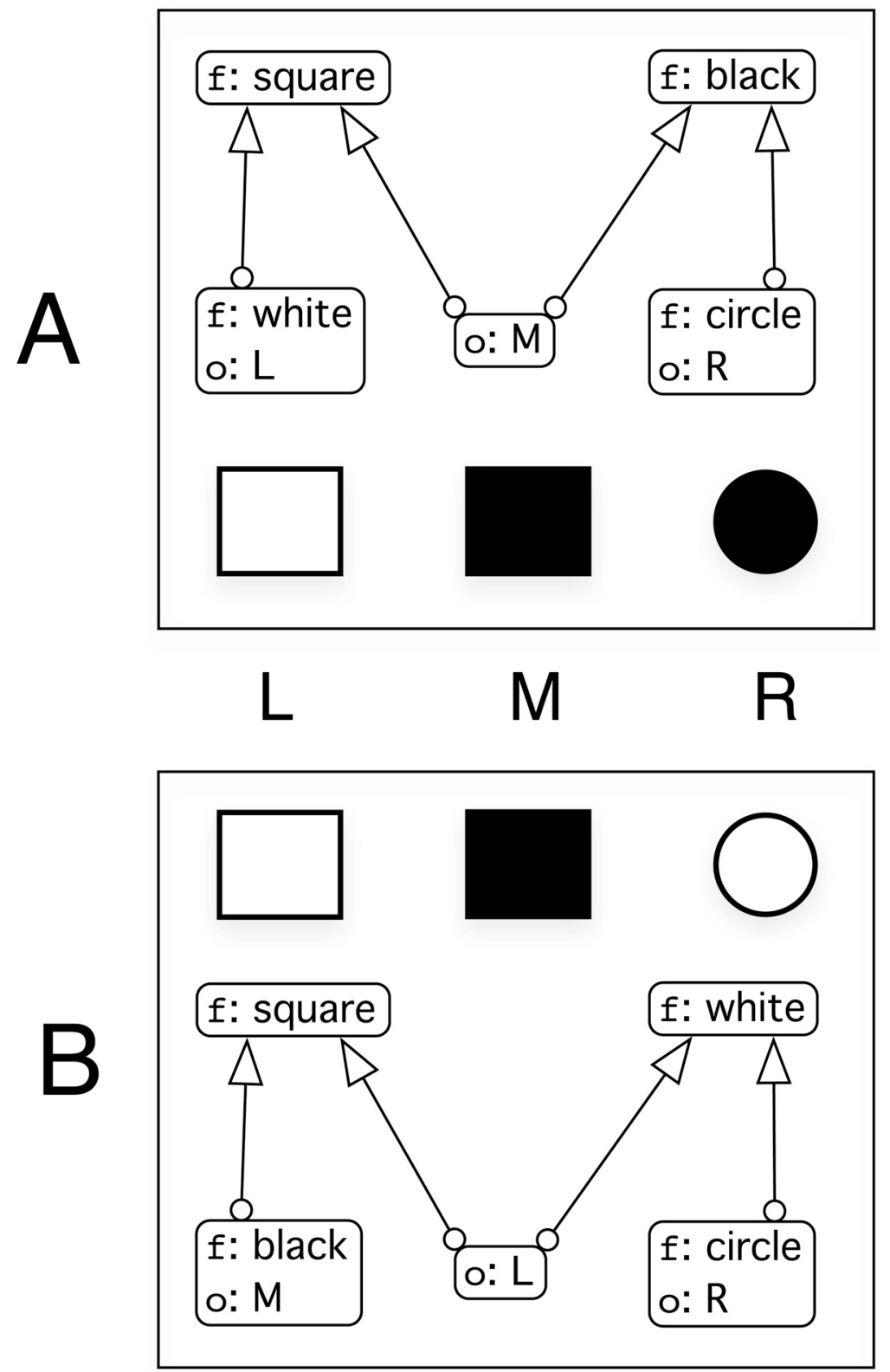

Figure 13. Galois lattices illustrating the effect of change of colour for the object on the right. 
change the saliency of the different properties and hence the saliency of the objects. A property, which distinguishes an object from the others and makes the object the only one of its species, is highlighted and makes the object "pop out", generating the well-known pop-out effect (Treisman \& Gelade, 1980). The property black is highlighted in b instead of white in a. We shall show how the formalisation of the context effect by the Galois lattice mechanism nicely explains how a given property of a category is made salient in a given context and another one in another context.

Suppose that the label "Tomato" is presented with the labels of the 14 food items which were judged as being fruit by the 10 subjects in the HéronBénaïcha (1998) experiment. We shall retain the description of the 14 kinds of fruit plus the description of the tomato for the properties for which there is a consensus between the 10 participants. The resulting Galois lattice is presented in Figure 14a: at each node the number of items (fruit including tomatoes), having the property corresponding to the node is indicated, the nodes in black being the description of the tomato. We can see that tomatoes have two properties which are specific: is [/eaten/cooked] and [/is eaten/in a meal/starter]. We can expect that those properties are made salient and will be given priority if the task is to describe a tomato among the other items. The other properties of tomatoes are shared properties of fruit and should not be made so salient.
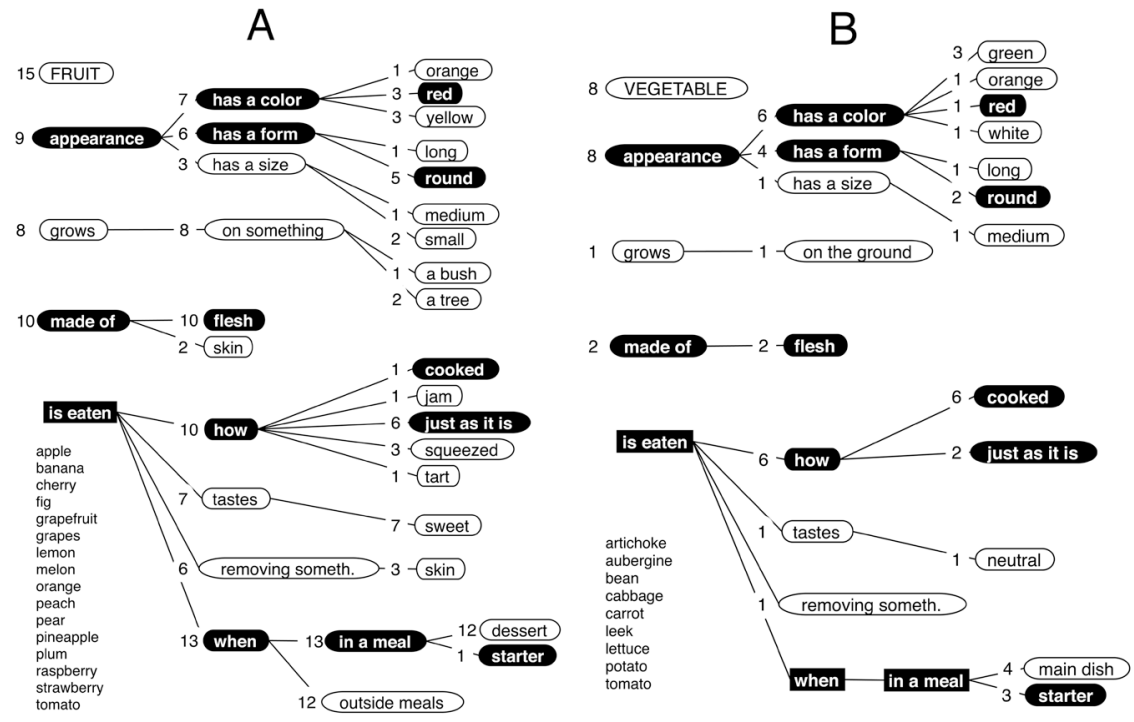

Figure 14. Description of the tomato (in black) among (A) fruit and (B) vegetables. 
Consider now the item tomato in the context of the seven items considered to be vegetables by all subjects. In the Galois lattice presented in Figure 14b, it appears that the tomato is unique among vegetables in terms of one property, red: this property is expected to be salient. In that manner different properties become salient in function of the context. Thus the Galois lattice can be used as a mechanism to explain why the same item may be attributed one property in a given context and another one in another context, and accordingly, why its category may change from a context to another.

The Galois lattice selection mechanism may generate powerful predictions concerning the effects of context. To exemplify this aspect, we shall consider the conditions of the property generation task as studied by Vrignaud (1998). Two conditions were tested: the usual one in which a single category is given for description and the second where two categories are presented simultaneously. In the case of an isolated category (say cabbage or apple), this theory predicts that the properties which will be evoked will be on one hand the most specific category to which the item belongs, i.e., more likely the base-level category (fruit or vegetable); and on the other hand the properties that are specific to the item and which differentiate the item from the others in the category: for cabbage (is green, is used for sauerkraut ...) for apple (is red and yellow, is peeled, is used to make cider ...). This is exactly what was observed by Héron-Bénaïcha (1998). The properties most often cited were first those regarding appearance (mainly colour), and those relative to the parts of the object (mainly what is eaten, the flesh or the leaves, or those where something must be removed for eating, the skin, stones, or pips). These are differentiating properties, whereas the other properties cited most often were very specific properties such as the consistency of flesh, the properties of skin (thick, thin, smooth, rough), the various types of preparation, the nutritive value, the dietetic particularities, the country it comes from, etc.

Consider now the condition where categories are presented in pairs. Suppose that we are interested by the properties of oranges paired with leeks, then paired with carrots. We assume that the description, which gave the properties recognised by all the subjects in the Héron-Bénaïcha experiment, is a reasonable approximation of the cognitive content of these categories in long-term memory. We shall compute which properties will be made salient when the item orange is presented with each of the two vegetables and, assuming that these properties will be produced in the property generating task, we shall compare the two lists and determine how many properties are shared. We will show that the theory predicts that more common properties will be attributed to the orange when it is presented in the context of a vegetable. 
Let us begin with the pair orange-leek. The most specific category, which includes the orange and leek (or carrot), is food. We retain all property lines that are present in the description of both items, i.e., [/grows], [/appearance], [/is eaten]. On each line we retain all the properties encountered until a stem is found which differentiates orange from leek, and we add the food subcategory fruit and vegetable, present in the description. The lattice is presented in Figure 15a (and the isomorphic binary relation in Table 3a): it shows both the similarities and the differences between the orange and leek. We are concerned with the differences, since they determine which properties will be made salient. These differences, which appear on the leaves of the
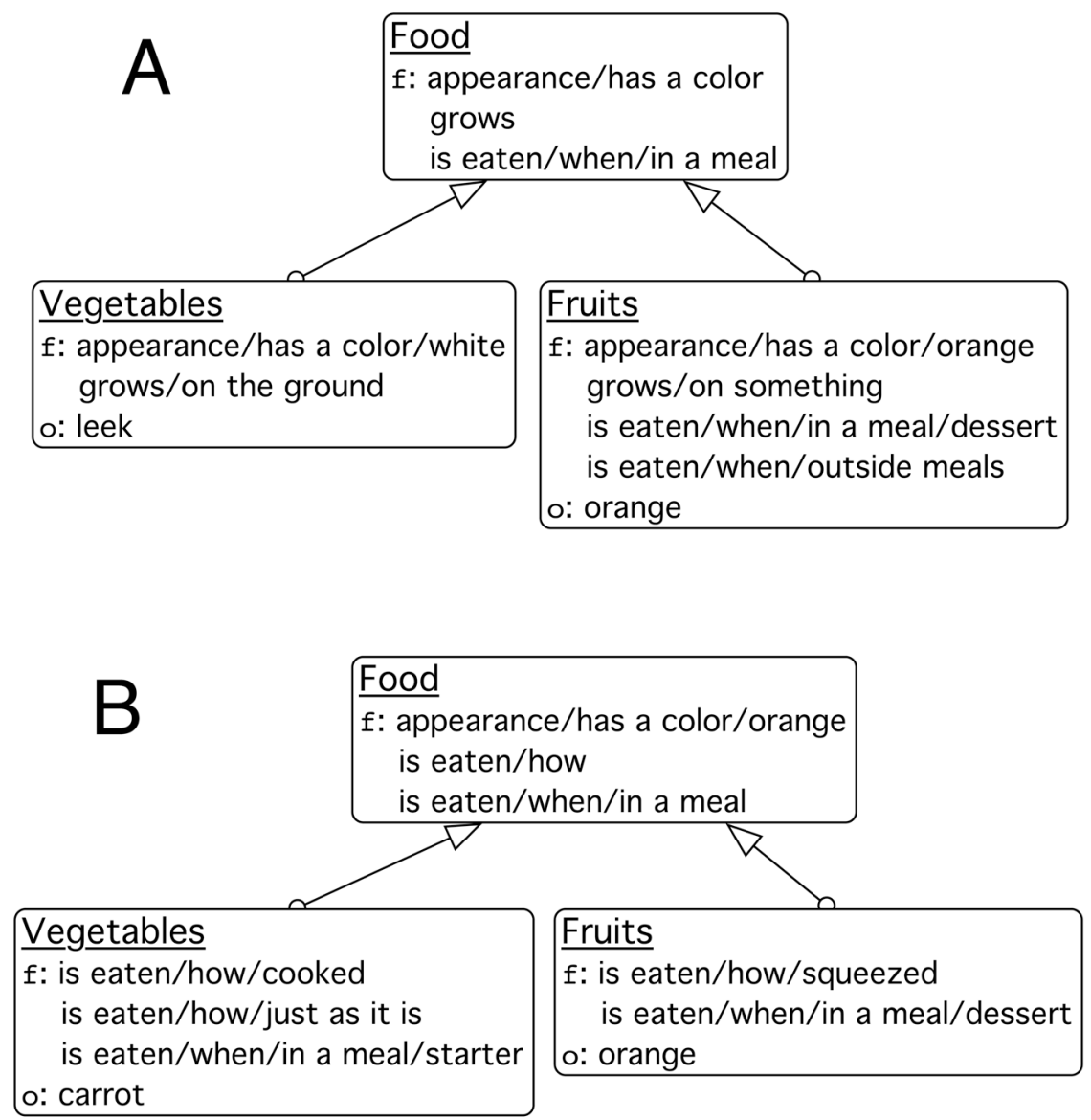

Figure 15. Commonalities and differences between orange and leek (A) and between orange and carrot (B). 
TABLE 3

Binary relations $a$ and $b$ isomorphic to graphs in Figure 15

\begin{tabular}{|c|c|c|c|c|c|}
\hline \multicolumn{4}{|c|}{ Leek Orange } & \multicolumn{2}{|c|}{ Carrot Orange } \\
\hline $\begin{array}{l}\text { is eaten/when/in a meal/dessert } \\
\text { appearance/has a colour/white }\end{array}$ & 1 & 1 & $\begin{array}{l}\text { is eaten/when/in a meal/entry } \\
\text { is eaten/when/in a meal/dessert }\end{array}$ & 1 & 1 \\
\hline appearance/has a colour/orange & & 1 & is eaten $/$ how $/$ cooked & 1 & \\
\hline grows/on the ground & 1 & & is eaten $/$ how $/$ just as it is & 1 & \\
\hline grows/on something & & 1 & is eaten/how/squeezed & & 1 \\
\hline & & & appearance/has a colour/orange & 1 & 1 \\
\hline
\end{tabular}

lattice, are: [/is food/a fruit], [/appearance/colour/orange], [/grows/on something/a tree], [/is eaten in a meal/dessert], [/is eaten/outside meals].

Similarly, in the graph of Figure $15 \mathrm{~b}$ and the corresponding binary relation of Table $3 b$, the properties, which will be highlighted for oranges when presented with carrots, are visible: [/is food/a fruit], [/is eaten/how /squeezed], [/is eaten in a meal/dessert]. Notice that the property appears in the context of carrot but not with leek. This is because not every participant attributed the property [/is eaten/how] to leeks. Conversely, [/grows on something/a tree] appears with leek but not with carrot, because [/grows/on the ground] is present for leek but not for carrot. The details may be left aside, because the supposed description in memory is very rough, but the important idea to retain is that the cognitive content of categories in memory is as important as context in determining which properties will be made salient. With the presented lattices there are properties that are highlighted in one or the other context and would supposedly be produced spontaneously for the orange: [/is food/a fruit], [/appearance/colour /orange], [/grows/on something], [/is eaten/how/squeezed], [/is eaten/outside meals], [/is eaten in a meal/dessert]. As most of these properties are also properties of fruits, they will be given in the same way for other exemplars of fruits presented in pairs with a vegetable, so that a fairly large number of properties will be common to all exemplars. In contrast we may expect that when the orange is presented in isolation, the only property that would be given on different occasions or by different subjects would be [/is food/a fruit], because the very specific properties are numerous and highly variable from one subject to another, and thus are not likely to be produced simultaneously. As we have reported, Vrignaud (1998) found the agreement about the content of a category was much more important when the subcategories were presented in the context of another category.

Briefly, the properties that are made salient are those that are in a property line common to both items and which are in contrast to the properties of the other item. The more different the items of the context are, the more general properties will be highlighted. We have previously 
proposed the idea that the larger the context the more accessible the general properties of a category will be, and we suggested that a classification task would produce a larger context than a property generation task. This idea could be supported in a more formal way using the Galois lattice approach. Additional assumptions would be necessary to do so for a classification task, because in such a task, due to the constraint of producing exclusive classes, compromises have to be made between the properties that are elicited. Some properties have to be neglected in this case, but it is a priori possible.

Let us now summarise and discuss implications of our theory. Our basic claim is that a single mechanism, the Galois lattice coupled with a definition of properties as property lines, explains both the formation categories in long-term memory and ad hoc categorisation. Categories arise when collections of objects happen to possess the same subset of properties, i.e., when groupings of properties are stable across contexts. These groupings are candidates to receive a name, given some constraints, such as to divide the objects into exhaustive and mutually exclusive groups, termed the mutual exclusivity assumption in the context of learning to name the objects (Markman, 1990), a principle that affords economy in thought and communication. Hence not all cognitive categories receive a name: there are hybrid categories that may be designated by different names of categories, depending on what properties are meant in the context. This is the basis of cross-classification and typicality effects. Naming facilitates the stabilisation in memory as well as communication.

As the categories that we consider are based on the description of objects, the theory applies to categories that are classes of objects and are designated by count names. Categories that are based on relations between objects (e.g., sister) deserve a more complex treatment, because the properties to be considered have to be defined on couples of objects, and are out of the scope of the present paper, as are those that deal with relations between complex objects (e.g., cause seen as a relations between two events).

The contexts in which we meet things every day are highly variable: these are not seen with the same other things, the goals in which they are involved differ, etc. The effect of the specific context is modelled as well by the Galois lattice mechanism that makes salient the similarities and differences between the objects that are present. As a consequence, the properties that will characterise the object will depend on the other objects in the context. The rule is that only the properties that are sufficient to characterise commonalities and differences of the objects will be used and then made salient. Hence, the larger is the number of objects present, the higher the number of properties necessary for the description and the higher the constraints on the properties to be used. It is for that reason that we predict a higher level of agreement when categories are presented by pairs and still higher when a group of category labels are presented for classification. The 
agreement will be the best in the judgement task, since the properties to be considered are given and are not to be retrieved.

The idea that a property is defined by a range of degrees of refinement and not only in terms of attribute and value gives sense to very abstract distinctions and intuitions not necessarily expressed in language. Obviously attributes share more or less resemblance: form, size, and colour appear more similar between them than localisation: they refer to the appearance of objects and they seem to be intrinsic to the object, while localisation expresses a relation between the object and other ones, and is extrinsic. Infants have the notion that two objects cannot be at the same place, as well as the distinction between animate and inanimate, the notion of support, etc. (Carey \& Spelke, 1994). These notions are not derived from linguistic categories but perceived as very basic properties of objects. Property lines are derived from the combination of innate capacities, of development, learning, and acquisition of language.

An original aspect of our proposal is that the same basic mechanism is responsible for various aspects of categorisation. This aspect has to be discussed in relation with the idea that categorisation has many uses, as has been stressed by Markman and Ross (2003). They point out that there are many uses of categories outside classification: inference, communication. They insist on the fact that information used in the category is not the same in an inference task and in a categorisation task. Although both tasks are isomorphic, if it is assumed that the label is regarded as a feature (in a classification task the label has to be guessed given the set of features; in an inference task the missing feature has to be guessed, given the label and the other features), it is not the case that the label is a mere feature. Yamauchi and Markman (2000) have compared both tasks with categories varying in similarity. The interesting case is the low-similarity condition: in that case the test stimulus in the inference task has the category label and one feature consistent with the corresponding category, but has three features which are characteristic of the opposite category. In this task participants tend to respond as a function of the category label, while in the classification task they are influenced by the similarity information.

This result is consistent with the prediction we could make from our theory. As we said, the category label stands for the set of correlated properties characteristic of the concept. The context of the inference task is the label and other features: the participant is expected to rely on the label rather than on the other features, because the label alone tells which attributes are relevant, and the other features are either irrelevant, or redundant with the label (in case of correlated attributes). By this reasoning, if a participant is informed that a pineapple is a fruit and asked how it grows, he will answer the typical way of growing for fruits, i.e., on a tree, which we have effectively observed. Suppose however that a pineapple is presented for 
classification with other fruits: a plum, an orange, a lemon, a pear, cherries etc. We may expect that the properties made salient in that context will be those that are different from the other fruits: it will appear big, while the other are small or medium, it will be the only one that has leaves. As a consequence, it will not be grouped with other fruits and it will be allotted to a separate class. In our view, a pineapple will be treated as an atypical fruit in a classification task, but as a typical fruit in an inference task.

Let us now examine how the mechanism we propose may explain how categories are used for communication. They are used mainly for two purposes. The first is to attribute a property to an entity using a category label. It has been shown that when asked by their child, mothers use either the subcategory label (robin) or the category label (bird) for typical examples, but only the subcategory label for atypical examples (turkey) (Labrell \& Boutet-Blouin, 2000; White, 1982). Although we differ from the typicality view, we have the same response as the supporters of this conception would probably give, but for another reason: since atypical examples are hybrid classes, which may be referred to several categories, it would be necessary to give the name of each of these categories, which would be confusing. A more specific prediction may be made for the designation of a particular object in a scene, which is the second objective in communication. Consider the example given in Figure 13: The Galois lattice selective mechanism makes salient which properties are unique and which are shared. If a property of an object is unique, the name of this property is sufficient for designating it in the context. For example, the form of the lefthand graph (a) in Figure 13 may be called "the white one", while in graph b the same form has to be called "the white square": white is a unique property of the left object in graph a, but is a shared property (with form) in graph $b$. These differences are due uniquely to the context. A similar idea has been proposed by Olson (1970).

\section{DISCUSSION: COMPARISON WITH RELATED PROPOSALS}

\section{The prototype view}

The aim of the paper is to contest the widely accepted idea that there is no consensus about the content of natural categories and the implied thesis that the assignment of an object to a category is based on the similarities of this object with the prototypes of the category, so that membership to a category is a matter of family resemblance (Rosch, 1975a; Rosch \& Mervis, 1975). Our claim is first that the data produced to support this thesis proceed from a single experimental situation, that these data are particular to the context of that task, and that other tasks reveal a high level of 
consensus. We propose a theory, based on the redefinition of what a property is and the modelling of the effect of context, which explains why such differences are generated between the experimental tasks. We argue that the cognitive content of natural categories is highly stable in long-term memory, but that all known properties are not necessarily accessed in a particular context. Only those that are relevant are considered and this produces variability in categorisation.

Otherwise we accept many of the ideas developed by Rosch and her coauthors: the important notion of typicality, the idea that categories are produced to simplify reality and that typical examples of a category provide the cognitive content of the category. We propose a reinterpretation of typicality effects. Atypical examples or subcategories correspond to objects or species with properties that allow them to be assigned to several contrasting categories. For instance, atypical examples of fruit and vegetables are hybrid categories (e.g., vegetables in one respect and fruit in another) which tend to be incorporated into the categories of typical fruit and typical vegetables, because there is a quasi-implication between the ways in which fruit and vegetables grow and are eaten. If we consider the network formed by the relations between cognitive categories, it would appear that the structure of this network is hierarchical but complex, as some categories have properties that are shared by several supercategories. For simplicity's sake, cultures tend to build taxonomies that are, at least locally, tree-like. Each category is subdivided into an exhaustive set of subcategories that are exclusive of each other, and names are then given to each one. Hybrid categories are not retained in these hierarchies and are not given specific names, because they are not compatible with this classification device and, besides, it is of no practical importance. Although they do not possess all the properties of the categories, they may be designated by the names of both contrasting categories. Due to the context, there will be no ambiguity as regards which properties are meant by the names of the categories. As we have shown, in a farming context, pineapples will be considered as a vegetable and tomatoes as fruit. In the context of choosing the menu for a meal, pineapples will be considered as fruit and tomatoes as vegetables. The context selects the properties that are relevant, since these properties are shared by the other elements of the context.

To conclude, hybrid categories are atypical because they possess only some of the properties of typical categories, even though they can be given the same label. Typicality effects are due to the assignment of names to categories: they do not imply that category boundaries are fuzzy. Cognitive categories are well defined, their content being constituted by the set of properties shared by typical examples. However, category names of typical categories may be extended to hybrid categories that have no specific name. The claim that very few properties are shared by all the exemplars of a given 
category has strongly boosted the idea that typicality effects are proof of category heterogeneity, and has helped to disqualify theories that set out to define the categories by conceptual content. We have shown that this claim, although widely accepted, is unfounded.

To summarise, our claim is that cognitive categories are quite stable, but that the meaning of lexical terms used to refer to them may be variable. The differences of accessibility in memory between typical and atypical exemplars of a same category from the set of characteristic properties evoked by its name are due to the fact that typical exemplars perfectly match the cognitive content of the category, while atypical exemplars may be referred not only to this category but also to an alternative category, because they possess the properties of both categories. Still, in a given mental context there is no ambiguity about which category they are intended to refer to. Namely, the context constrains the properties to be considered, making the properties of the relevant category more salient.

\section{The classic view}

There are two basic ideas in the classic view: categories have a well-defined conceptual content, and membership of an object to a category is decided on the basis of a set of necessary and sufficient conditions, by checking that all the properties defining the category are present. We accept the first thesis but not the second, because it assumes that the decision for membership is independent of the context. Now we have insisted on the ubiquity of the flexibility of categorisation and on the flexibility of the meaning assigned to a label as being a function of the context. The properties we consider when we assign an object to a category are rarely the same and not all properties of the category are considered, because, as we have shown, the context reduces the properties to be considered to those that are most informative and relevant in the context, and we are not a position to check exhaustively the defining properties. In the habitual practice of categorisation, we are led to take the context into account, so that the idea of verification of truth conditions is untenable.

This is the reason why it is practically impossible to produce definitions of natural categories: their main function is not definitional. Reference to a category is used to state a set of properties in an economical way, thereby avoiding the chore of listing them all individually. It is used to give a description of an entity, not to assess whether the entity really falls within the extension of the class. It should be noted that when the extension of a category has to be accurately stated, as in the case of laws and regulations, precise definitions are produced so that disputes do not concern the limits of the category. Instead they focus on whether or not the case in point possesses the critical properties of the category as defined by the law. This 
is precisely the case when categorisation has to be independent of the context.

Let us consider for example the notion of ownership. In everyday language we do not distinguish between different forms of possession, so when somebody says I have a nice flat, it is not understood whether s/he owns or rents it. What is meant is that the flat $\mathrm{s} /$ he lives in is nice. While a single word is enough in daily life, several are used in law, corresponding to explicit distinctions. Property is distinguished from use: one may own property and have the use of it, or own property and not have the use of it, (bare ownership). Usage only, or bare ownership, or both, may be transferred. Things become more complex still when non-material objects are involved, such as literary property. Nevertheless, legal definitions are precise enough to enable judges to decide whether or not the case brought before them represents plagiary.

\section{The alignment view}

The property line approach is obviously related to the alignment view, although quite different in scope. This theory states that structural alignment, first invoked to explain analogy making (Gentner, 1983), is a very general process which is at work in comparison (Markman \& Gentner, 1993a , 1993b, 2000), as a determiner of similarity (Medin et al., 1993). It may be able to promote abstraction, acting "as a bridge by which similaritybased processes can give rise to abstract rules" (Gentner \& Medina, 1998, p.266). Structural alignment is defined as a search for the deepest possible level of correspondence between two representations. The result of the search is a commonality at the deepest level and differences at the lower levels are said to be alignable because they correspond to elements put into correspondence in the matching process. A structurally consistent alignment must satisfy three rules: one-to-one mapping (each element in a representation has its correspondent in the other), parallel connectivity (if the predicates match, the arguments must also match), and systematicity (among matching systems a preference is given to the matching system with higher-order constraining relations).

Although the property-line approach we propose differs from alignment theory in premises (the nature of relations constituting the hierarchy are not the same) as well as in scope (we do not intend to explain analogy making or comparison but categorisation in context), both theories share the idea that there are clusters of connected properties organised in a hierarchy which have a major importance in processing similarities as well as differences between objects. When properties organised in such a hierarchy are present in a set of objects in such a way that a commonality appears at a given level of the hierarchy and differences at the lower levels, these commonalities and 
differences are privileged in that they become more salient. No matter whether these hierarchies are based on a property line or on a structural match, the consequence is the same: the contrasting point in the hierarchy with a commonality above and a difference below makes the commonality as well as the differences more salient. We suggest that it is a consequence of the idea common to both theories that there are interconnected properties that are organised into hierarchic clusters. In our view these relations are semantic implications stored in memory. In the alignment theories they are constructed on-line by a structural alignment process, but the nature of the relations behind the hierarchy has no importance for this process. For this reason we make similar predictions regarding the effect of context on the differential highlighting of the properties of an object depending on the other object presented with it. The fact that these contrast effects are observed in immediate perception (Treisman \& Gelade, 1980) and in comparison situations (Bowdle \& Gentner, 1997; Gentner \& Gunn, 2001; Gentner \& Medina, 1998; Markman \& Gentner, 1993a, 1993b, 2000) is consistent with the idea that the relations between the properties in the hierarchy may be either stored in memory or constructed on-line in the course of an elaborated interpretation process. We conclude that the property-line approach, although fundamentally different in scope from the structural alignment theory, makes similar predictions on the effect of context on perceived similarities, which depart from the common assumption of independence of features.

We may be more specific in comparing our approach with the alignment theory regarding studies that have extended this view to the study of natural categories. Markman and Wisniewski (1997) have applied the distinction of alignable vs non-alignable differences to the definition of basic-level categories. Categories at this level of categorisation are recognised as highly differentiated: they maximise the similarity within the category and the dissimilarity with contrasting categories. These authors have questioned this view in a series of experiments where participants are required to produce commonalities and differences for pairs of categories belonging to different levels: superordinate level, basic level with the same or a different superordinate, subordinate level categories having either the same basic category, or a different basic category with the same superordinate or a different basic category with a different superordinate. It appears that basic categories differ in a different way from superordinate categories: they have both many commonalities and many differences, while superordinate ones have few commonalities and many non-alignable differences. Subordinate categories have a large number of commonalities as well as alignable differences even if they are not derived from the same basic category, provided that they belong to the same superordinate category. This is the 
proof that categories coming from a common superordinate have a common abstract structure.

We have an interpretation that is similar in some respects an different in another. In our view alignable differences correspond to the point where a specification appears in the property line: it is a difference with a commonality just above. It happens that the superordinate level corresponds to a general level of a property and that it is only at the next level (the basic level) that it is specified how the property is physically realised. For instance, the superordinate category of vehicles has properties like: way of moving, type of support for moving, used for transportation etc. It is only at the basic level that it is specified how each property is realised: the vehicle is driven by a motor or an animal, it may move on road, railway, snow, it is used for private or public transportation. These specifications define different basic-level categories: bus, train, car, and sledge. The characteristic of the basic level is that this specification appears for many properties at the same time. This is not far from what Markman and Wisniewski say. Our position is slightly different as regards non-alignable differences. In our view, non-alignable differences also correspond to commonalities (because any difference implies a commonality), but these commonalities are more abstract and not so salient. A vehicle is a kind of thing used by humans, which is similar to other artefacts used for other functions. There are similarities between functions as well as between natural things. Superordinate categories like clothing and jewellery are probably seen as similar to each other and dissimilar from fruit, vegetables, and food, and in the last group there are obvious inclusion relations. These similarities correspond to higher levels of generality of the property, so that there are several nodes between the difference and the closest commonality. We do not see the distinction as a dichotomy but rather as a matter of degree: in other words a difference is more or less alignable depending how many nodes in the property tree separate the difference from the closest communality.

Another distinction related to the concept of alignment has to be discussed: the distinction between consistent and inconsistent contrast proposed by Billman and Davila (2001) in the field of concept learning. Given a set of contrastive categories, a contrast is said to be consistent if the categories have a common set of relevant attributes and differ only at the level of the values of these attributes. The contrast is said to be inconsistent when the categories do not have the same relevant attributes.

In the example given by the authors, categories are constituted by fictive animals described by five attributes, two of them being relevant and perfectly correlated: sound, movement, habitat, colour, head and limbs with three values each. In the consistent contrast the correlated attributes are sound and move, and the categories vary by their value: croak and fly for category 1 , yodel and slide for category 2 , buzz and bounce for category 3 . 
In the inconsistent contrast condition attributes 1 and 2 are relevant for category 1, attributes 3 and 4 for category 2, attributes 5 and 6 for category 3: each attribute is relevant for one category, so that no attribute can be discarded. Consistent contrast categories are much easier to learn, especially when in the inconsistent contrast condition the value of an irrelevant attribute is allowed to be the correct value of a relevant attribute for another category. Moreover the correlation between the two attributes is more
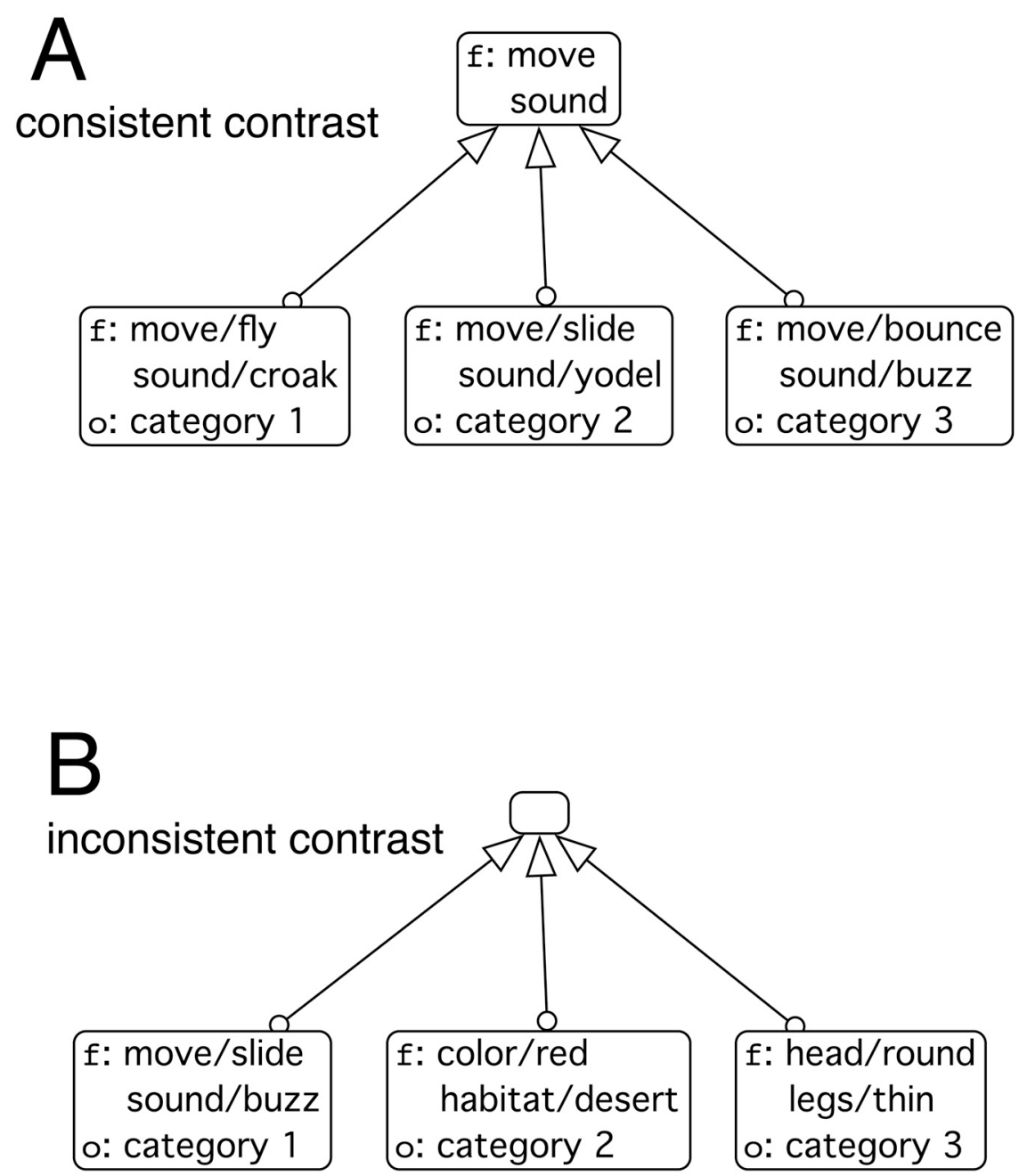

Figure 16. Galois lattices illustrating the conditions of consistent (A) and inconsistent (B) contrast. 
frequently remarked in the consistent contrast condition. Finally if in the same condition participants are given a single example of a new category and are tested for learning this category, they respond on the basis of the attributes that were relevant for the previously learned categories and not on the basis of the similarity to the example.

The authors argue that selectivity takes place at two levels: the attribute values as relevant to a particular category and attributes as such which are common to the three categories. If we apply the Galois lattice approach, the two conditions are described by the graphs of Figure 16 that present the description of objects corresponding to perfect learning. As it is made clear by graph $\mathrm{A}$, what has to be learnt in the consistent contrast condition is a property defined at two levels: at the general level the property is common to the three categories (all produce a sound and move); at the specific level the categories are differentiated by the specification of the type of sound and of movement. In the case of inconsistent contrast, what is common is a property that is much more general, the fact that each category has two features. As there is a great distance between the commonality and the difference, it is much more difficult to remark and will not help learning. In the consistent condition there are also two common properties, but these two are specified as being sound and move. Obviously, the distinction between consistent and inconsistent contrast is an extension of the distinction between alignable and non-alignable differences.

Billman and Davila rightly refer to attention models formerly developed in the framework of discrimination learning where attentional learning modulates rule discovery. Several models have been proposed (Bower \& Trabasso, 1964; Lovejoy, 1966; Richard, 1974; Zeaman \& House, 1963). In those models the reinforcement operates at two levels: the level of the association of the response to a given value of the attribute and the level of the attention to the attribute (or its salience). They predict that an intradimensional reversal shift is more difficult for animals than an extradimensional shift at moderate levels of learning but that the reverse is true after overlearning. They would predict the type of results obtained by Billman and Davila. We may remark that the property line approach provides a straightforward interpretation of this process: the reinforcement acts as an activation process, which propagates along the property line with a parameter of decay.

\section{The flexibility view}

For the flexibility viewpoint we are referring to the thesis developed by Barsalou in a series of influential studies demonstrating the instability of categories (see Barsalou, 1987, 1989; Barsalou \& Medin, 1986, for a summary) and claiming that there is no conceptual core (Barsalou, 1993a, 1993b; Jones \& Smith, 1993). As we have developed empirical and 
theoretical arguments in favour of the idea that categories have a welldefined conceptual content, we certainly disagree with this claim. However there are a number of important points of convergence between our position and Barsalou's theory.

First we completely agree with the idea of the importance of the variability of the content of the categories as a function of the context. The basic role of categories is not taxonomic. Whether they are used for communicating, for predicting information about an object, or for accomplishing a specific goal, all these functions are highly dependent on the context. Hence the content of the categories, which is relevant to the context, may differ. We agree on the idea that the temporary conceptualisations that are highly dependent on the context are based on knowledge stored in long-term memory, and that this knowledge is highly stable. As we have noted earlier, Barsalou (1993a) was the first author to demonstrate the differences observed in a judgement task in contrast to those observed in property generation tasks and to signal the high level of agreement as regards the attribution of a feature to a category in the judgement task. As he says, "Viewing concepts as temporary constructs by no means implies that people do not have well-established knowledge." Instead this view does assume the presence of relatively stable knowledge in long-term memory (Barsalou, 1989, p. 94). What Barsalou claims, is that the knowledge stored in memory for a category is not controlling behaviour: if it were so, there would not be such a variability in performance in attribution of category membership or category definitions.

Our main point of disagreement with Barsalou's position is that knowledge in memory related to categories is highly organised. It provides a frame of description for objects in the form of a property-lines tree and contains a definition of natural categories as subsets of correlated properties in a property-line format. We propose a theory correlating which effect of context is produced by a Galois lattice mechanism and this explains how differences in specific category content are generated by the differences in mental and physical contexts. This mechanism is crucial to explain how variability in categorisation activity is compatible with the existence of stable structures in long-term memory. We want to stress that the organisation in memory we propose concerns not only the structure of categories but also primarily the organisation of the coding system implied in the description of the objects. In the property-lines approach the higher levels are abstract but they are related to the lower levels so that no dissociation is assumed between the perceptual and conceptual levels, and a continuum may even be conceived between these levels. We have not investigated the perceptual aspects of categories that would constitute the most specific parts of property lines but we do not deny their existence. Our approach seems compatible with the theory developed by Barsalou that 
conceptual processing is grounded on perceptual systems (Barsalou 1993a, 1999; Barsalou \& Prinz, 1997, Goldstone \& Barsalou, 1998).

\section{The conceptual coherence view}

Our position is compatible with the theory-based view, as defined by Ross and Spalding (1994). In actual fact, we agree with many ideas put forward in this theory. Natural categories are based on what people think makes things what they are, not on what things are in fact. The representations that people have are often simplifications that are not verified in each case but which allow clear-cut distinctions to be made between categories and increase the coherence of the cognitive system. The goal of categorisation is not to provide definitions but to predict non-visible properties or to convey information about some property meant by the speaker. In a communication context, it does not matter whether some properties are missing in some exemplars, because another way of expressing the target property will be used if the name of category is in any way misleading. For example, if somebody shows pineapples growing in a field to a child and wants to explain what they are, s/he will probably not say that they are fruit, as s/he would for cherries, but instead will say that they are eaten as a dessert. Moreover, we completely agree with the idea that although similarity plays a role, it does not form the basis of category assignment. Surface properties are constrained by deeper ones, so that we only retain surface properties that are consistent with deep ones-because appearances are usually not deceiving (Murphy \& Medin, 1985). Much of our energy has been devoted to making the difference between surface properties and deep ones more precise and to highlighting several relations that contribute to this distinction and form the basis of conceptual coherence.

It would take too long to discuss how relations of an empirical nature such as causal relations or relations of function to organ contribute to conceptual coherence, so we will limit ourselves to semantic implication. It is fundamental to our understanding of what a property is and it defines a specificity-generality dimension. This dimension introduces a first type of constraint. Specific properties are constrained by more general ones, in that the absence of a general property excludes every specific property that implies it. For instance, if an entity has no size, as is the case for uncountable entities like bread (some bread) as opposed to a slice of bread (which is an individual entity), it definitely has no length, width, or height, because length, width, and height are specifications of size for objects having specific shapes. Moreover, if an entity has a general property, it necessarily has some more specific ones. For example, if an entity is movable, it is certainly not the case that it is neither self-movable nor transportable. Constraints exist not only along a property line but also between different property lines. 
Assuming that the set of properties describing a domain constitute a treelike structure, the tree defines distances between the properties. A simple measure of distance is the length of the shortest path leading from one to another within the tree. The distance between properties introduces strong constraints between properties that may account for the intuitive idea that two particular properties are closer than two other ones. If told about two unknown entities, one that walks and another that crawls, and then about two more, one that walks and another that swims, the first ones are deduced to be more similar than the second ones.

Although many disputes have arisen over this relation (Fellbaum \& Miller, 1990; Rips \& Conrad, 1989, 1990), the implication relation has never before been considered in this systematic way, probably for two reasons. On the one hand, categories have been considered almost exclusively from the extensional point of view, which makes the relation between the classes a relation of inclusion. They have practically never been considered from the intensional point of view, where the relation between classes is a relation of implication between the properties. Instead, attention has been focused on linguistic categories (verbs vs nouns) rather than cognitive ones (properties vs categories).

Categories are not to be confused with properties, because even though they are used for descriptive purposes in the same way as properties, categories are derived from the empirical relations that appear when different types of objects are described in the same description space. The stable empirical associations observed in objects generate categories. However, there may exist semantic relations of an inclusion type between categories (Collins \& Quillian, 1969), which reflect semantic implications between their properties.

\section{CONCLUSION}

The assignment of objects to categories is based on properties. Properties are lines of features linked by a relation of implication and distributed along a generality/specificity dimension, such as [/appearance/colour/red/vermilion]. When taken together, two properties lines will have segments in common and segments that are specific. The least general feature, common to both properties, constitutes a node where the two property lines diverge. If we consider that the root of all property lines is a node with the trivial meaning is a property, any set of properties is a tree providing a domain of description for objects. Thus, the description of any object constitutes a sub-tree of a domain tree.

Categories are based on another type of relation of an empirical nature that appears when the descriptions of large sets of entities are joined together. They are developed via a categorisation process in the following 
way: categorisation consists of building the semantic network generated by the description of the objects present in the context. In this network, only the least specific properties, which are sufficient to distinguish the objects present in the situation, and the least general properties, which are sufficient to cover the whole set, are selected. The network presents the empirical relations that exist between the objects in the situation. The nodes show which properties are associated and the links represent empirical implications. The nodes at the bottom represent the properties that are specific, the other nodes those properties that are shared. This network is unstable, as it depends on the particular objects that are present. It represents ad hoc categories that are relevant for the context and which can be used in that context to designate the objects. Ad hoc categorisation is a fundamental process based on a cognitive economy principle: only those properties that are the most informative are taken into account. This principle has also been shown to be basic to communicative interactions (Sperber \& Wilson, 1986).

Categories are formed with associations that remain stable across different contexts. Many cognitive categories have no name but may be designated by periphrases. The name of a category is used as shorthand for the set of properties associated within the category. This set is assumed to be stable and constitutes the category's semantic content. The name of a category activates every property characteristic of that category. It should be emphasised that what is stable is the conceptual content of the category, and that there will be a degree of variability in the specific sense of the word when interpreted in a given context. Saying "he is an eagle" does not convey the same meaning as "this is an eagle": the selection of features that are relevant to the context allows for the metaphorical use of words that is fundamental in language. Selection according to context operates in the same way for properties evoked by the name of a category as for directly perceived categories.

Categories may be defined at more or less specific levels, due to the fact that they are made up of properties that are themselves considered at more or less specific levels. For instance, mammals, fish, and birds are regarded as animals because they move on their own. They are considered to be living creatures because they feed themselves, reproduce, and have a lifecycle like plants, which, on the other hand, do not move. Mammals, birds, and fish differ among other things in their mode of reproduction and in the environment in which they move. Mammals differ in their way of moving (walking or jumping, using four or two legs).

Our theory proposes a mechanism that can account both for the variability of the scope of verbal category labels and for the stability of cognitive structures which allow us to reason about objects by applying inferences drawn from their known properties to new ones. Variability is due to the categorisation process, which always takes place in a given context. Stability, on the other hand, is related to knowledge structures stored in 
long-term memory, which interact with the objects present in the context to generate the semantic network relevant to that particular context. The stability of cognitive structures is necessary if we are to explain the variability brought about by the context concerning the meaning of category labels. A major part of the controversy about the existence of a conceptual core is due to neglecting the effect of context in the specific task used to investigate the conceptual content of categories. Once this effect is recognised, it appears that the existence of a conceptual core is not incompatible with the fact that variability is prevalent in some tasks, since this variability is reduced and even disappears in tasks involving a larger semantic context.

As in other positions such as Barsalou's theory, we claim that context generates variability in categorisation, but with the qualification that the variability in the conceptual content of the category is more or less important, depending on the extent of this content, which is activated by the context. A narrow context generates variability, but when context provides a large and even complete coverage of the category, the variability is reduced or even vanishes. A good illustration is the case of category-based induction, where a property attributed to subcategories of a common category is generalised to this category. According to the prediction that confidence in the generalised inference is a function of the coverage of the category by the subcategories, Osherson, Smith, Wilkie, Lopez, and Shafir (1990) showed that the less the premises are similar, the stronger is the argument. As a consequence, the variability is not so pervasive as it was claimed and the context may produce stability as well as variability. Variability is manifested mainly in interpretation phenomena, while stability is characteristic of category-based inductive and deductive inference.

Manuscript received 5 January 2004 Revised manuscript received 3 June 2004

\section{REFERENCES}

Barbut, M., \& Monjardet, B. (1970). Ordre et classification: algèbre et combinatoire. Paris: Hachette.

Barsalou, L. W. (1983). Ad hoc categories. Memory and Cognition, 11, 211-227.

Barsalou, L. W. (1987). The instability of graded structure: Implications for the nature of concepts. In U. Neisser (Ed.), Concepts and conceptual development. New York: Cambridge University Press.

Barsalou, L. W. (1989). Intraconcept similarity and its implications for interconcept similarity. In S. Vosniadou \& A. Ortony (Eds.), Similarity and analogical reasoning (pp. 76-121). New York: Cambridge University Press.

Barsalou, L. W. (1993a). Structure, flexibility, and linguistic vagary in concepts: Manifestations of a compositional system of perceptual symbols. In A. C. Collins, S. E. Gathercole, M. A. Conway, \& P. E. Morris (Eds.), Theories of memory (pp. 29-101). Hove, UK: Lawrence Erlbaum Associates Ltd. 


\section{6}

Barsalou, L. W. (1993b). Challenging assumptions about concepts. Cognitive Development, 8 , $169-180$.

Barsalou, L. W. (1999). Perceptual symbol systems. Behavioral and Brain Sciences, 22, 577-609.

Barsalou, L. W., \& Medin, D. L. (1986). Concepts: Static definitions or context-dependent representations? Cahiers de Psychologie Cogntive, 2, 187-202.

Barsalou, L. W., \& Prinz, J. J. (1997). Mundane creativity in perceptual symbol systems. In T. B. Ward, S. M. Smith, \& J. Vaid (Eds.), Conceptual structures and processes: Emergence, discovery and change. Washington, DC: American Psychological Association.

Belleza, F. S. (1984). Reliability of retrieval from semantic memory: Common categories. Bulletin of the Psychonomic Society, 22, 324-326.

Bernard, J. M., \& Poitrenaud, S. (1999). L'analyse implicative bayesienne multivariée d'un questionnaire binaire: Quasi-implications et treillis de Galois simplifié. Mathématiques, Informatique, Sciences Humaines, 147, 25-46.

Billman, D., \& Davila, D. (2001). Consistent contrast aids concept learning. Memory and Cognition, 29, $1022-1035$.

Bowdle, B. F., \& Gentner, D. (1997). Informativity and asymmetry in comparisons. Cognitive Psychology, 34, 244-286.

Bower, G. H., \& Trabasso, T. (1964). Concept identification. In R. C. Atkinson (Ed.), Studies in mathematical psychology (pp. 32-94). Stanford, CA: Stanford University Press.

Carey, S., \& Spelke, E. (1994). Domain-specific knowledge and conceptual change. In L. A. Hirschfeld \& S. A. Gelman (Eds.), Mapping the mind: Domain specificity in cognition and culture (pp. 169-200). New York: Cambridge University Press.

Chauveix, S., \& Egoroff, C. (2003). Etablissement d'une liste de descripteurs sensoriels: Une nouvelle approche basée sur la catégorisation. Psychologie Française, 48, 79-89.

Collins, A. Q., \& Quillian, M. R. (1969). Retrieval time from semantic memory. Journal of Verbal Learning and Verbal Behavior, 8, 240-247.

Fellbaum, C., \& Miller, G. A. (1990). Folk psychology or semantic entailment? Comment on Rips and Conrad (1989). Psychological Review, 97, 565-570.

Gelman, S. A., \& Heyman, G. D. (1999). Carrot-eaters and creature-believers: The effects of lexicalization on children's inferences about social categories. Psychological Science, 10, $489-492$.

Gentner, D. (1983). Structure mapping: A theoretical framework for analogy. Cognitive Science, 7, $155-170$.

Gentner, D., \& Gunn, V. (2001). Structural alignment facilitates noticing of differences. Memory and Cognition, 29, 565-577.

Gentner, D., \& Medina, J. (1998). Similarity and the development of rules. Cognition, 65, $263-$ 297.

Goldstone, R. L., \& Barsalou, L. W. (1998). Reuniting perception and conception. Cognition, $65,231-262$.

Greenberg, J. (1966). Language universals, with special reference to feature hierarchies. The Hague: Mouton.

Grice, H. P. (1975). Meaning. Philosophical Review, 66, 377-388.

Guénoche, A., \& Van Mechelen, I. (1993). Galois approach to the induction of concepts. In I. Van Mechelen, J. Hampton, R. S. Michalski, \& P. Theuns (Eds.), Theoretical views and inductive data analysis (pp. 287-308). New York: Academic Press.

Hampton, J. (1993). Conceptual combination. In K. Lamberts \& D. Shanks (Eds.), Knowledge, concepts and categories (pp. 133-159). Hove, UK: Psychology Press.

Héron-Bénaicha, G. (1998). Etude des activités de catégorisation. Mémoire de Maîtrise, Université de Paris VIII. 
Hubert, L., \& Arabie, P. (1994). The analysis of proximity matrices through sums of matrices having (anti-)Robinson forms. British Journal of Mathematical and Statistical Psychology, 47, $1-40$.

Jones, S. S., \& Smith, L. B. (1993). The place of perception in children's concepts. Cognitive Development, 8, $113-139$.

Labrell, F., \& Boutet-Blouin, C. (2000). Dénomination et description parentales des catégories d'objets en direction de l'enfant de 4 ans. Psychologie Francaise, 45(2), $177-186$.

Lopez, A., Atran, S., Coley, J. D., \& Medin, D. L. (1997). The tree of life: Universal and cultural features of folkbiological taxonomies and inductions. Cognitive Psychology, 32, $251-295$.

Lovejoy, E. (1966). Analysis of the overlearning reversal effect. Psychological Review, 73, 87 103.

Malt, B. C. (1995). Category coherence in cross-cultural perspective. Cognitive Psychology, 29, $85-148$.

Malt, B. C., Sloman, S. A., Gennari, S., Shi, M., \& Wang, Y. (1999). Knowing versus naming: Similarity and the linguistic categorization of artifacts. Journal of Memory and Language, $40,230-262$.

Markman, E. M. (1990). Constraints children place on word meaning. Cognitive Science, 14(1), [Special Issue] $57-77$.

Markman, A. B., \& Gentner, D. (1993a). Splitting the differences: A structural alignment view. Journal of Memory and Language, 32, 517-535.

Markman, A. B., \& Gentner, D. (1993b). Structural alignment during similarity comparisons. Cognitive Psychology, 25, $431-467$.

Markman, A. B., \& Gentner, D. (2000). Structure mapping in the comparison process. American Journal of Psychology, 113(4), 501-538.

Markman, A. B., \& Ross, B. H. (2003). Category use and category learning. Psychological Bulletin, 129(4), $592-613$.

Markman, A. B., \& Wisniewski, E. J. (1997). Similar and different: The differentiation of basiclearning categories. Journal of Experimental Psychology: Leaning, Memory and Cognition, 23, $54-70$.

Medin, D. L., Goldstone, R. L., \& Gentner, D. (1993). Respects for similarity. Psychological Review, 100, 254-278.

Medin, D. L., Lynch, E. B., Coley, J. D., \& Atran, S. (1997). Categorisation and reasoning among tree experts: Do all roads lead to Rome? Cognitive Psychology, 32, 49-96.

Medin, D. L., \& Ortony, A. (1989). Psychological essentialism. In S. Vosniadou \& A. Ortony (Eds.), Similarity and analogical reasoning (pp. 179-195). New York: Cambridge University Press.

Murphy, G. L., \& Medin, D. L. (1985). The role of theories in conceptual coherence. Psychological Review, 92, 289-316.

Osherson, D. N., Smith, E. E., Wilkie, O., Lopez, A., \& Shafir, E. (1990). Category-based induction. Psychological Review, 97, 185-200.

Poitrenaud, S. (1995). The PROCOPE semantic network: An alternative to action grammars. International Journal of Human-Computer Studies, 42, 31-69.

Politzer, G. (1991). L'informativité des énoncés: Contraintes sur le jugement et le raisonnement. Intellectica, 11, 11-147.

Richard, J. F. (1974). Attention et apprentissage. Paris: Presses Universitaires de France.

Rips, L. J. (1989). Similarity, typicality and categorisation. In S. Vosniadou \& A. Ortony (Eds.), Similarity and analogical reasoning (pp. $21-59$ ). New York: Cambridge University Press.

Rips, L. J., \& Conrad, F. G. (1989). Folk psychology of mental activity. Psychological Review, 96, $187-207$. 


\section{8}

Rips, L. J., \& Conrad, F. G. (1990). Parts of activities: Reply to Fellebaum and Miller. Psychological Review, 97, 571-575.

Rosch, E. (1975a). Cognitive reference points. Cognitive Psychology, 7, 532-547.

Rosch, E. (1975b). Cognitive representations of semantic categories. Journal of Experimental Psychology, 104(3), 192-233.

Rosch, E., \& Mervis, C. B. (1975). Family resemblances: Studies in the internal structure of categories. Cognitive Psychology, 7, 573-605.

Rosch, E., Mervis, C. B., Gray, W. D., Johnson, D. M., \& Boyes-Braem, P. (1976). Basic objects in natural categories. Cognitive Psychology, 8, 382-439.

Ross, B. H., \& Murphy, G. L. (1999). Food for thought: Cross-clarification and category organization in a complex real-world domain. Cognitive Psychology, 38, 495-553.

Ross, B. H., \& Spalding, T. L. (1994). Concepts and categories. In R. J. Sternberg (Ed.), Thinking and problem solving (pp. 119-148). New York: Academic Press.

Sperber, D., \& Wilson, D. (1986). Relevance: Communication and cognition. London: Blackwell.

Storms, G., Van Mechelen, I., \& De Boeck, P. (1994). Structural analysis of the intension and extension of semantic concepts. European Journal of Cognitive Psychology, 6, 43-75.

Tourangeau, R., \& Rips, L. (1991). Interpreting and evaluating metaphors. Journal of Memory and Language, 30, $452-472$.

Treisman, A. M., \& Gelade, G. (1980). A feature integration theory of attention. Cognitive Psychology, 12, 97-136.

Tversky, A. (1977). Features of similarity. Psychological Review, 84, 327-352.

Tversky, B., \& Hemenway, K. (1984). Objects parts and categories. Journal of Experimental Psychology: General, 113, 172-191.

White, T. G. (1982). Naming practices, typicality and underextension in child language. Journal of Experimental Child Psychology, 33, 324-336.

Wille, R. (1984). Line diagrams of hierarchical concept systems. International Classification, 2 , $77-86$.

Vrignaud, P. (1998). Approche différentielle de la typicalité. Thèse de Doctorat de l'Université de Paris V.

Wittgenstein, L. (1953). Philosophical investigations. New York: Macmillan.

Yamauchi, T., \& Markman, A. B. (2000). Inference using categories. Journal of Experimental Psychology, Learning, Memory and Cognition, 26, 776-795.

Zeaman, D., \& House, B. J. (1963). The role of attention in retardate discrimination learning. In M. R. Ellis (Ed.), Handbook of mental deficiency (pp. 159-223). New York: McGraw-Hill. 